Publ. RIMS, Kyoto Univ.

40 (2004), 91-123

\title{
An Inverse Problem for an Elliptic Equation
}

\author{
By \\ Robert DALMASSO*
}

\begin{abstract}
We consider the following overdetermined boundary value problem: $\Delta u=-\lambda u-$ $\mu$ in $\Omega, u=0$ on $\partial \Omega$ and $\frac{\partial u}{\partial n}=\psi$ on $\partial \Omega$, where $\lambda$ and $\mu$ are real constants and $\Omega$ is a smooth bounded planar domain. A very interesting problem is to examine whether one can identify the constants $\lambda$ and $\mu$ from knowledge of the normal flux $\frac{\partial u}{\partial n}$ on $\partial \Omega$ corresponding to some nontrivial solution. It is well known that if $\Omega$ is a disk then such identification of $(\lambda, \mu)$ is completely impossible. Some partial results have already been obtained. The purpose of this paper is to extend and to improve these results. Moreover we also examine the interesting case where $\psi$ is constant.
\end{abstract}

\section{$\S 1$. Introduction}

Consider the elliptic boundary value problem

$$
\begin{gathered}
\Delta u=-\lambda u-\mu \text { in } \Omega, \\
u=0 \text { on } \partial \Omega,
\end{gathered}
$$

where $\lambda$ and $\mu$ are real constants and $\Omega$ is a smooth bounded planar domain. An interesting problem is to examine whether one can identify the constants $\lambda$ and $\mu$ from knowledge of the normal flux $\partial u / \partial n$ on $\partial \Omega$ corresponding to some nontrivial solution of (1.1)-(1.2). For more general right hand sides this inverse problem arises for instance in plasma physics in connection with the modelling of Tokamaks [2]. But even in the very particular case of an affine term the

Communicated by T. Kawai. Received November 11, 2002.

2000 Mathematics Subject Classification(s): 35J05, 35R30.

*Laboratoire LMC-IMAG, Equipe EDP, Tour IRMA, BP 53, F-38041 Grenoble Cedex 9, France.

e-mail: Robert.Dalmasso@imag.fr 
problem is difficult. It is well known that if $\Omega$ is a disk then such identification of $(\lambda, \mu)$ is completely impossible, even in the case where a sign is imposed on the right hand side of the equation: It is shown in [11] that there is a continuum of coefficient pairs $\left(\lambda, \mu_{\lambda}\right) \in \mathbb{R}^{2}$, and therefore a continuum of affine functions, which give rise to the same normal derivative on the boundary. We refer the reader to paper [11] for a more detailed description of the problem in general and the difficulties encountered.

A partial answer to this problem was first obtained by Vogelius in [11], and more recently we have also given a contribution [6].

We briefly describe the results obtained in [11]. In the case where a sign is imposed on the right hand side of the equation, $\Omega$ is a bounded, strictly convex $C^{3, \alpha}$ domain which is not a disk. Then it suffices to assume that the normal derivative is not identically zero to show that there exist at most finitely many pairs of coefficients. For the case of solutions without the sign condition imposed extra conditions on the domain and conditions on the normal derivative are needed. The normal derivative is not identically constant and it has at most countably many zeros. $\Omega$ is a bounded, convex $C^{3, \alpha}$ domain whose boundary curvature is only zero at a countable number of points. Moreover $\Omega$ is not of constant width and has the so-called Schiffer property.

The width of a convex planar domain in a given direction is the distance between two parallel supporting lines perpendicular to that direction. A set of constant width has the same width in all directions. Clearly disks have constant width. However there are plenty of smooth domains which have constant width but which are not disks: See [3], [8] and [11].

A simply connected $C^{2, \alpha}$ domain $\Omega(\alpha \in(0,1])$ is said to have the Schiffer property if (for any $\lambda$ ) the only solution to the overdetermined boundary value problem

$$
\begin{aligned}
\Delta v & =-\lambda v-\mu \quad \text { in } \Omega, \\
v & =0, \frac{\partial v}{\partial n}=0 \quad \text { on } \partial \Omega,
\end{aligned}
$$

is the trivial solution $v=0$ (corresponding to $\mu=0$ ). We always consider classical solutions. It is well known that disks do not have the Schiffer property. Indeed let $J_{z}$ denote the $z$-th Bessel function and let $r>0$ be such that $J_{1}(\sqrt{r})=0$. Then the function

$$
v_{r}(x)=\frac{1}{r}\left(\frac{J_{0}(\sqrt{r}|x|)}{J_{0}(\sqrt{r})}-1\right), x \in \Omega
$$

satisfies (1.3)-(1.4) with $\lambda=r$ and $\mu=1$ when $\Omega$ is the unit disk. 
The Schiffer conjecture asserts that in any dimension balls are the only $C^{2, \alpha}$ domains with connected boundaries for which (1.3)-(1.4) has a nontrivial solution for even a single value of $\lambda$.

If the above conditions are satisfied, then there exist at most finitely many pairs of coefficients.

In [6] we have also obtained a "finiteness" result, which is much more precise than in [11]. We have made some restrictions on the domain, but our assumption on the normal derivative is in a sense optimal: We assume that the normal flux is not identically constant. Indeed, we have the following wellknown conjecture (see Berenstein [1]): If (1.1)-(1.2) with $\mu=0$ has a nontrivial solution with constant normal derivative, is $\Omega$ a disk? Our method of proof is completely different. However any domain in the class that we consider in [6] has the Schiffer property and is not of constant width.

In the present paper, we shall first study the case of domains with constant width, for which the proof given in [11] fails. We first treat a particular class of domains of constant width. Then we shall consider still other classes of domains.

We need some notations and some definitions. When $\partial \Omega$ is at least $C^{2}$ we denote by $n=\left(n_{1}, n_{2}\right)$ the exterior normal and by $K$ the curvature. We first define $l: \partial \Omega \rightarrow \mathbb{R}$ by

$$
l(x)=x_{1} n_{2}(x)-x_{2} n_{1}(x) \quad x \in \partial \Omega .
$$

Now let $f: \partial \Omega \rightarrow \mathbb{R}$ be such that $f \in L^{2}(\partial \Omega)$, then we set

$$
d_{n}(f)=\frac{1}{2 \pi} \int_{\partial \Omega}\left(n_{1}(x)+i n_{2}(x)\right)^{n} f(x) d \sigma,
$$

for $n \in \mathbb{Z}$ and

$$
\begin{aligned}
k_{1}(f) & =2 \inf \left\{n \in \mathbb{N}^{*} ; d_{2 n+1}(1) d_{3}\left(f^{2}\right) \neq d_{2 n+1}\left(f^{2}\right) d_{3}(1)\right\}, \\
k_{2, r}(f) & =2 \inf \left\{n \geq r+1 ; d_{2 n+1}(K f) \neq 0\right\}-1, \quad r \in \mathbb{N}^{*}, \\
k_{3, r}(f) & =2 \inf \left\{n \geq 2(r+1) ; d_{2 n}(K f) \neq 0\right\}-1, \quad r \in \mathbb{N}^{*}, \\
k_{4}(f) & =2 \inf \left\{n \in \mathbb{N}^{*} ; d_{2 n}(l) d_{3}\left(f^{2}\right) \neq d_{2 n}\left(f^{2} l\right) d_{3}(1)\right\}, \\
k_{5}(f) & =2 \inf \left\{n \in \mathbb{N}^{*} ; d_{2 n}(l) d_{2}\left(f^{2} l\right) \neq d_{2 n}\left(f^{2} l\right) d_{2}(l)\right\},
\end{aligned}
$$

and

$$
k_{6}(f)=\inf \left\{n \in \mathbb{N}^{*} ; d_{2}(l) d_{2 n+1}\left(f^{2}\right) \neq d_{2 n+1}(1) d_{2}\left(f^{2} l\right)\right\} .
$$

(Notice that it may be the case that $k_{j}(f)=\infty$ ). 
Definition 1.1. $\Omega \subset \mathbb{R}^{2}$ is said to be of class $\mathcal{B}$ if the following conditions hold:

i) $\Omega$ is a bounded convex open set and $\partial \Omega$ is a $C^{\infty}$ curve with positive curvature $K$.

ii) $d_{3}(1) \neq 0$.

iii) $d_{2 n}(1)=0$ for $n \in \mathbb{Z}^{*}$.

iv) $A=\left\{n \in \mathbb{N}^{*} ; d_{2 n+1}(1) \neq 0\right\}$ is finite.

Remark 1. We shall show that any domain of class $\mathcal{B}$ is of constant width.

Let $\Omega$ be a strictly convex planar domain. Given $x \in \partial \Omega$, we denote by $x^{*} \in \partial \Omega \backslash\{x\}$ the only point such that the tangent lines to $\partial \Omega$ through $x$ and $x^{*}$ are parallel.

Now we can state our first results.

Theorem 1.1. Let $\Omega \subset \mathbb{R}^{2}$ be of class $\mathcal{B}$. Let $\psi \in C^{\infty}(\partial \Omega)$ be a real valued function and assume that $\psi$ is not identically constant and that

$$
\frac{\psi^{2}(x)}{K(x)} \neq \frac{\psi^{2}\left(x^{*}\right)}{K\left(x^{*}\right)}
$$

for some $x \in \partial \Omega$. Let $k_{0}$ be defined as follows:

1) If $k_{1}(\psi)<\infty$, then $k_{0}=k_{1}(\psi)$;

2) If $k_{1}(\psi)=\infty$, then $k_{2, m}(\psi)<\infty$ or $k_{3, m}(\psi)<\infty$ where $m=\max A$. If $k_{2, m}(\psi)<\infty$, then $k_{0}=k_{2, m}(\psi)$. If $k_{2, m}(\psi)=\infty$, then $k_{0}=k_{3, m}(\psi)$.

Then there exist at most $k_{0}$ different pairs of coefficients $\left(\lambda_{k}, \mu_{k}\right) \in \mathbb{R}^{2}$ such that the Cauchy problem

$$
\Delta u=-\lambda_{k} u-\mu_{k} \quad \text { in } \quad \Omega, \quad u=0 \quad \text { and } \quad \frac{\partial u}{\partial n}=\psi \quad \text { on } \quad \partial \Omega
$$

has a solution.

For domains of class $\mathcal{B}$ the inverse problem with a sign imposed on the right hand side is an easy consequence of Theorem 1.1. We have the following Corollary. 
Corollary 1.1. Let $\Omega \subset \mathbb{R}^{2}$ be of class $\mathcal{B}$. Let $\psi \in C^{\infty}(\partial \Omega)$ be a real valued function and assume that $(*)$ holds for some $x \in \partial \Omega$. Let $k_{0}$ be defined as follows:

1) If $k_{1}(\psi)<\infty$, then $k_{0}=k_{1}(\psi)$;

2) If $k_{1}(\psi)=\infty$, then $k_{2, m}(\psi)<\infty$ or $k_{3, m}(\psi)<\infty$ where $m=\max A$. If $k_{2, m}(\psi)<\infty$, then $k_{0}=k_{2, m}(\psi)$. If $k_{2, m}(\psi)=\infty$, then $k_{0}=k_{3, m}(\psi)$.

Then there exist at most $k_{0}$ different pairs of coefficients $\left(\lambda_{k}, \mu_{k}\right) \in \mathbb{R}^{2}$ such that the Cauchy problem

$$
\Delta u=-\lambda_{k} u-\mu_{k} \leq 0 \quad \text { in } \quad \Omega, \quad u=0 \quad \text { and } \quad \frac{\partial u}{\partial n}=\psi \quad \text { on } \quad \partial \Omega
$$

has a solution.

We say that a convex planar domain is locally of constant width if there exist $\xi_{0} \in S^{1}$ and an open neighborhood $U$ of $\xi_{0}$ in $S^{1}$ such that the width of $\Omega$ is constant in all directions $\xi \in U$.

Definition 1.2. $\Omega \subset \mathbb{R}^{2}$ is said to be of class $\mathcal{C}$ if the following conditions hold:

i) $\Omega$ is a bounded convex open set and $\partial \Omega$ is a $C^{\infty}$ curve with positive curvature $K$.

ii) $d_{3}(1) \neq 0$.

iii) There exists $n \in \mathbb{Z}^{*}$ such that $d_{2 n}(1) \neq 0$.

iv) $\Omega$ is not locally of constant width.

We have the following results.

Theorem 1.2. Let $\Omega \subset \mathbb{R}^{2}$ be of class $\mathcal{C}$. Let $\psi \in C^{\infty}(\partial \Omega)$ be a real valued function and assume that $\psi$ is not identically constant. Then $k_{1}(\psi)<\infty$ or $k_{4}(\psi)<\infty$ and if $k_{0}=\min \left(k_{1}(\psi), k_{4}(\psi)\right)$, then there exist at most $k_{0}$ different pairs of coefficients $\left(\lambda_{k}, \mu_{k}\right) \in \mathbb{R}^{2}$ such that the Cauchy problem (1.5) has a solution.

For domains of class $\mathcal{C}$ we have the following Corollary. 
Corollary 1.2. Let $\Omega \subset \mathbb{R}^{2}$ be of class $\mathcal{C}$. Let $\psi \in C^{\infty}(\partial \Omega)$ be a real valued function and assume that $\psi$ is not identically zero. Then $k_{1}(\psi)<\infty$ or $k_{4}(\psi)<\infty$ and if $k_{0}=\min \left(k_{1}(\psi), k_{4}(\psi)\right)$, then there exist at most $k_{0}$ different pairs of coefficients $\left(\lambda_{k}, \mu_{k}\right) \in \mathbb{R}^{2}$ such that the Cauchy problem (1.6) has a solution.

Definition 1.3. $\Omega \subset \mathbb{R}^{2}$ is said to be of class $\mathcal{D}$ if the following conditions hold:

i) $\Omega$ is a bounded convex open set and $\partial \Omega$ is a $C^{\infty}$ curve with positive curvature $K$.

ii) $\Omega$ is not locally of constant width.

iii) $d_{2}(l) \neq 0$.

Now we can state our last results.

Theorem 1.3. Let $\Omega \subset \mathbb{R}^{2}$ be of class $\mathcal{D}$. Let $\psi \in C^{\infty}(\partial \Omega)$ be a real valued function and assume that $\psi$ is not identically constant. Then $k_{5}(\psi)<\infty$ or $k_{6}(\psi)<\infty$ and if $k_{0}=\min \left(k_{5}(\psi), k_{6}(\psi)\right)$, then there exist at most $k_{0}$ different pairs of coefficients $\left(\lambda_{k}, \mu_{k}\right) \in \mathbb{R}^{2}$ such that the Cauchy problem (1.5) has a solution.

For domains of class $\mathcal{D}$ we have the following Corollary.

Corollary 1.3. Let $\Omega \subset \mathbb{R}^{2}$ be of class $\mathcal{D}$. Let $\psi \in C^{\infty}(\partial \Omega)$ be a real valued function and assume that $\psi$ is not identically zero. Then $k_{5}(\psi)<\infty$ or $k_{6}(\psi)<\infty$ and if $k_{0}=\min \left(k_{5}(\psi), k_{6}(\psi)\right)$, then there exist at most $k_{0}$ different pairs of coefficients $\left(\lambda_{k}, \mu_{k}\right) \in \mathbb{R}^{2}$ such that the Cauchy problem (1.6) has a solution.

In Section 2 we give some preliminary results. In Section 3 we first show that when $\Omega \subset \mathbb{R}^{2}$ is of class $\mathcal{B}$ then $\Omega$ is of constant width. Then we prove Theorem 1.1 and Corollary 1.1. In Section 4 we prove Theorem 1.2 and Corollary 1.2. Theorem 1.3 and Corollary 1.3 are proved in Section 5. We begin Section 6 with the following remark: we show that when $\Omega \subset \mathbb{R}^{2}$ is of class $\mathcal{B}$, $\mathcal{C}$ or $\mathcal{D}$ then $\Omega$ has the Schiffer property. Then we give three particular results in the case where $\psi$ is a non zero constant. Finally we conclude with some examples in Section 7. 


\section{§2. Preliminaries}

For $v \in C^{\infty}(\bar{\Omega})$ and $i \in\{1,2\}$ we write

$$
v_{j}=\frac{\partial v}{\partial x_{j}} .
$$

Throughout this Section $\Omega \subset \mathbb{R}^{2}$ denotes a bounded simply connected open set with $C^{\infty}$ boundary $\partial \Omega$ and $u \in C^{\infty}(\bar{\Omega})$ is a solution of problem (1.1)-(1.2) satisfying

$$
\frac{\partial u}{\partial n}=\psi \quad \text { on } \partial \Omega
$$

for some $\psi \in C^{\infty}(\partial \Omega)$, where $n=\left(n_{1}, n_{2}\right)$ is the exterior normal to $\partial \Omega$.

Let $x=x(s)=\left(x_{1}(s), x_{2}(s)\right), s \in[0, L]$, be a parametrization of $\partial \Omega$ by arc length. We denote by $\tau(s)=\left(\tau_{1}(s), \tau_{2}(s)\right)$ the tangent to $\partial \Omega$ at $x(s)$ and by $\nu(s)=\left(\nu_{1}(s), \nu_{2}(s)\right)$ the exterior normal to $\partial \Omega$ at $x(s)$. We have

$$
\tau_{1}(s)=x_{1}^{\prime}(s), \quad \tau_{2}(s)=x_{2}^{\prime}(s) \quad s \in[0, L],
$$

and

$$
\nu_{1}(s)=x_{2}^{\prime}(s), \quad \nu_{2}(s)=-x_{1}^{\prime}(s) \quad s \in[0, L] .
$$

The Frenet formulas are

$$
x^{\prime \prime}(s)=-\kappa(s) \nu(s), \quad \nu^{\prime}(s)=\kappa(s) x^{\prime}(s) \quad s \in[0, L],
$$

where $\kappa=\kappa(s)$ is the curvature.

For any function $f: \partial \Omega \rightarrow \mathbb{R}$, we define $\tilde{f}=f \circ x$.

Let $p \geq 1$ be an integer. We define

$$
\varphi(p)=\sum_{j=0}^{p}\left(\begin{array}{l}
p \\
j
\end{array}\right) i^{j} \frac{\partial^{p} u}{\partial x_{1}^{p-j} \partial x_{2}^{j}} .
$$

Lemma 2.1. We have

$$
\varphi(1)_{2}+i \varphi(1)_{1}=-i(\lambda u+\mu)
$$

and for $p \geq 1$ we have

$$
\begin{aligned}
\varphi(p+1)_{2}+i \varphi(p+1)_{1} & =-i \lambda \varphi(p) \\
\varphi(p+1) & =\varphi(p)_{1}+i \varphi(p)_{2} .
\end{aligned}
$$

Proof. The proof follows from elementary calculation. 
Lemma 2.2. We have

$$
\begin{aligned}
& \tilde{\varphi}(1)=\left(\nu_{1}+i \nu_{2}\right) \tilde{\psi} \\
& \tilde{\varphi}(2)=-\mu\left(\nu_{1}+i \nu_{2}\right)^{2}+2 i\left(\nu_{1}+i \nu_{2}\right) \tilde{\varphi}(1)^{\prime},
\end{aligned}
$$

and for $p \geq 1$

$$
\tilde{\varphi}(p+2)=-\lambda\left(\nu_{1}+i \nu_{2}\right)^{2} \tilde{\varphi}(p)+2 i\left(\nu_{1}+i \nu_{2}\right) \tilde{\varphi}(p+1)^{\prime} .
$$

Proof. Since $u=0$ on $\partial \Omega$, we have

$$
u_{j}=n_{j} \frac{\partial u}{\partial n}=n_{j} \psi \quad \text { on } \partial \Omega
$$

from which we get $\tilde{\varphi}(1)$. Now we have

$$
\tilde{\varphi}(p)^{\prime}=-\tilde{\varphi}(p)_{1} \nu_{2}+\tilde{\varphi}(p)_{2} \nu_{1} .
$$

Using Lemma 2.1 we deduce that

$$
\begin{aligned}
& \tilde{\varphi}(1)_{1}=i\left(\nu_{1}+i \nu_{2}\right)\left(\tilde{\varphi}(1)^{\prime}+i \mu \nu_{1}\right), \\
& \tilde{\varphi}(1)_{2}=i\left(\nu_{1}+i \nu_{2}\right)\left(-i \tilde{\varphi}(1)^{\prime}+i \mu \nu_{2}\right),
\end{aligned}
$$

and for $p \geq 1$

$$
\begin{aligned}
& \tilde{\varphi}(p+1)_{1}=i\left(\nu_{1}+i \nu_{2}\right)\left(\tilde{\varphi}(p+1)^{\prime}+i \lambda \nu_{1} \tilde{\varphi}(p)\right), \\
& \tilde{\varphi}(p+1)_{2}=i\left(\nu_{1}+i \nu_{2}\right)\left(-i \tilde{\varphi}(p+1)^{\prime}+i \lambda \nu_{2} \tilde{\varphi}(p)\right) .
\end{aligned}
$$

The result follows from (2.3), (2.4) and Lemma 2.1.

Lemma 2.3. Let $k \geq 3$ be an integer.

1) If $k=2 p+1$ we have

$$
\tilde{\varphi}(k)=\left[(-1)^{p} \lambda^{p} \tilde{\psi}+\mu \sum_{j=1}^{p} a_{j, k} \lambda^{p-j}+\sum_{j=1}^{p} b_{j, k} \lambda^{p-j}\right]\left(\nu_{1}+i \nu_{2}\right)^{k},
$$

in $[0, L]$, where $a_{j, k}$ and $b_{j, k}$ are independent of $\lambda$ and $\mu$. Moreover

$$
a_{1, k}=(-1)^{p-1} p(k+1) \kappa .
$$

2) If $k=2 p$ we have

$$
\tilde{\varphi}(k)=\left[(-1)^{p} \lambda^{p-1} \mu+\mu \sum_{j=2}^{p} c_{j, k} \lambda^{p-j}+\sum_{j=1}^{p} d_{j, k} \lambda^{p-j}\right]\left(\nu_{1}+i \nu_{2}\right)^{k},
$$


in $[0, L]$, where $c_{j, k}$ and $d_{j, k}$ are independent of $\lambda$ and $\mu$. Moreover

$$
d_{1, k}=(-1)^{p-1} i k\left(\tilde{\psi}^{\prime}+p i \kappa \tilde{\psi}\right) .
$$

3) Finally we have:

3a) If $k=2 p+1$

$$
b_{1, k}=2 i \sum_{j=1}^{p}(-1)^{p-j}\left(d_{1,2 j}^{\prime}+2 i j \kappa d_{1,2 j}\right)
$$

where $d_{1,2}=2 i\left(\tilde{\psi}^{\prime}+i \kappa \tilde{\psi}\right)$.

3b) If $k=2 p$

$$
c_{2, k}=2 i \sum_{j=1}^{p-1}(-1)^{p-j-1}\left(a_{1,2 j+1}^{\prime}+(2 j+1) i \kappa a_{1,2 j+1}\right) .
$$

Proof. The proof will be by induction on $k$. Assume first that $k=3$. Using Lemmas 2.1 and 2.2 we obtain

$$
\tilde{\varphi}(3)=-\left(\lambda \tilde{\psi}-4 \mu \kappa+8 i \kappa\left(\tilde{\psi}^{\prime}+i \kappa \tilde{\psi}\right)+4\left(\tilde{\psi}^{\prime}+i \kappa \tilde{\psi}\right)^{\prime}\right)\left(\nu_{1}+i \nu_{2}\right)^{3},
$$

hence $a_{1,3}=4 \kappa$.

If $k=4$, using what we have just proved and Lemma 2.2 we get

$\tilde{\varphi}(4)=\left(\lambda \mu+2 i \mu\left(a_{1,3}^{\prime}+3 i \kappa a_{1,3}\right)-4 i \lambda\left(\tilde{\psi}^{\prime}+2 i \kappa \tilde{\psi}\right)+2 i\left(b_{1,3}^{\prime}+3 i \kappa b_{1,3}\right)\right)\left(\nu_{1}+i \nu_{2}\right)^{4}$ hence $d_{1,4}=-4 i\left(\tilde{\psi}^{\prime}+2 i \kappa \tilde{\psi}\right)$.

Now assuming that the result holds for $k \geq 4$, we shall prove that it holds for $k+1$. We have two cases to consider.

Case 1: $k=2 p$ with $p \geq 2$. Using the induction hypothesis, Lemma 2.2 and (2.1) we obtain

$$
\begin{aligned}
\tilde{\varphi}(k+1)= & {\left[(-1)^{p} \lambda^{p} \tilde{\psi}-\mu \sum_{j=1}^{p-1} a_{j, k-1} \lambda^{p-j}-\sum_{j=1}^{p-1} b_{j, k-1} \lambda^{p-j}\right.} \\
& +2 i\left(i k \kappa(-1)^{p} \lambda^{p-1} \mu+\mu \sum_{j=2}^{p}\left(c_{j, k}^{\prime}+i k \kappa c_{j, k}\right) \lambda^{p-j}\right. \\
& \left.\left.+\sum_{j=1}^{p}\left(d_{j, k}^{\prime}+i k \kappa d_{j, k}\right) \lambda^{p-j}\right)\right]\left(\nu_{1}+\nu_{2}\right)^{k+1} .
\end{aligned}
$$


Therefore

$$
a_{1, k+1}=(-1)^{p-1} p(k+1) \kappa .
$$

Case 2: $k=2 p+1$ with $p \geq 2$. Using the induction hypothesis, Lemma 2.2 and (2.1) we obtain

$$
\begin{aligned}
\tilde{\varphi}(k+1)= & {\left[(-1)^{p+1} \lambda^{p} \mu-\mu \sum_{j=2}^{p} c_{j, k-1} \lambda^{p+1-j}-\sum_{j=1}^{p} d_{j, k-1} \lambda^{p+1-j}\right.} \\
& +2 i\left((-1)^{p} \lambda^{p}\left(\tilde{\psi}^{\prime}+i k \kappa \tilde{\psi}\right)+\mu \sum_{j=1}^{p}\left(a_{j, k}^{\prime}+i k \kappa a_{j, k}\right) \lambda^{p-j}\right. \\
& \left.\left.+\sum_{j=1}^{p}\left(b_{j, k}^{\prime}+i k \kappa b_{j, k}\right) \lambda^{p-j}\right)\right]\left(\nu_{1}+\nu_{2}\right)^{k+1} .
\end{aligned}
$$

Therefore

$$
d_{1, k+1}=(-1)^{p} i(k+1)\left(\tilde{\psi}^{\prime}+(p+1) i \kappa \tilde{\psi}\right) .
$$

Now we can prove 3 ). From the proof above it follows that

$$
b_{1,3}=2 i\left(d_{1,2}^{\prime}+2 i \kappa d_{1,2}\right),
$$

and for $p \geq 2$

$$
b_{1,2 p+1}=2 i\left(d_{1,2 p}^{\prime}+2 i p \kappa d_{1,2 p}\right)-b_{1,2 p-1},
$$

from which we deduce $3 \mathrm{a}$ ). Now we have

$$
c_{2,4}=2 i\left(a_{1,3}^{\prime}+3 i \kappa a_{1,3}\right),
$$

and for $p \geq 3$

$$
c_{2,2 p}=2 i\left(a_{1,2 p-1}^{\prime}+(2 p-1) i \kappa a_{1,2 p-1}\right)-c_{2,2 p-2},
$$

and $3 \mathrm{~b}$ ) follows.

Lemma 2.4. For all $w \in C^{2}(\bar{\Omega})$ satisfying

$$
\Delta w+\lambda w=0 \quad \text { in } \Omega
$$

we have

$$
\int_{\partial \Omega} w \psi d \sigma+\mu \int_{\Omega} w d x=0
$$


Proof. Using Green's formula we can write

$$
\begin{aligned}
\lambda \int_{\Omega} w u d x & =-\int_{\Omega} u \Delta w d x \\
& =-\int_{\Omega} w \Delta u d x+\int_{\partial \Omega} w \psi d \sigma \\
& =\lambda \int_{\Omega} w u d x+\mu \int_{\Omega} w d x+\int_{\partial \Omega} w \psi d \sigma
\end{aligned}
$$

and the lemma follows.

\section{Lemma 2.5.}

1) We have

$$
\int_{\partial \Omega} \psi^{2}\left(x_{1} n_{2}-x_{2} n_{1}\right) d \sigma=0 .
$$

2) We have

$$
\begin{aligned}
& \lambda \int_{0}^{L} \tilde{\psi}^{2}\left(x_{1} \nu_{2}-x_{2} \nu_{1}\right)\left(\nu_{1}+i \nu_{2}\right)^{2} d s \\
& +\mu^{2} \int_{0}^{L}\left(x_{1} \nu_{2}-x_{2} \nu_{1}\right)\left(\nu_{1}+i \nu_{2}\right)^{2} d s+A \mu+B=0
\end{aligned}
$$

where $A$ and $B$ are independent of $\lambda$ and $\mu$. Moreover

$$
A=2 i \int_{0}^{L} \tilde{\psi}\left(2 \kappa\left(x \cdot \nu+i\left(x_{1} \nu_{2}-x_{2} \nu_{1}\right)\right)-1\right)\left(\nu_{1}+i \nu_{2}\right)^{2} d s .
$$

3) Let $k=2 p$ with $p \geq 2$. We have

$$
\begin{aligned}
0= & (-1)^{p} \lambda^{p-1}\left[\lambda \int_{0}^{L} \tilde{\psi}^{2}\left(x_{1} \nu_{2}-x_{2} \nu_{1}\right)\left(\nu_{1}+i \nu_{2}\right)^{k} d s\right. \\
& \left.+\mu^{2} \int_{0}^{L}\left(x_{1} \nu_{2}-x_{2} \nu_{1}\right)\left(\nu_{1}+i \nu_{2}\right)^{k} d s\right]+\mu^{2} \sum_{j=2}^{p} A_{j, k} \lambda^{p-j} \\
& +\mu \sum_{j=1}^{p} B_{j, k} \lambda^{p-j}+\sum_{j=1}^{p} C_{j, k} \lambda^{p-j}
\end{aligned}
$$

where $A_{j, k}, B_{j, k}$ and $C_{j, k}$ are independent of $\lambda$ and $\mu$. Moreover

$$
B_{1, k}=(-1)^{p} i k \int_{0}^{L} \tilde{\psi}\left(\kappa\left(2 x \cdot \nu+i k\left(x_{1} \nu_{2}-x_{2} \nu_{1}\right)\right)-1\right)\left(\nu_{1}+i \nu_{2}\right)^{k} d s .
$$


Proof. 1) Taking $w=x_{1} u_{2}-x_{2} u_{1}$ in Lemma 2.4 and integrating, we get

$$
\int_{\partial \Omega} \psi^{2}\left(x_{1} n_{2}-x_{2} n_{1}\right) d s=0
$$

2) Take $w=x_{1} \varphi(2)_{2}-x_{2} \varphi(2)_{1}$ in Lemma 2.4. Using (2.4) with $p=1$ and integrating we get

$$
\begin{aligned}
0= & \mu \int_{0}^{L}\left(x_{1} \nu_{2}-x_{2} \nu_{1}\right) \tilde{\varphi}(2) d s-\lambda \int_{0}^{L} \tilde{\psi}^{2}\left(x_{1} \nu_{2}-x_{2} \nu_{1}\right)\left(\nu_{1}+i \nu_{2}\right)^{2} d s \\
& +\int_{0}^{L} \tilde{\psi}\left(x \cdot \nu+i\left(x_{1} \nu_{2}-x_{2} \nu_{1}\right)\right) \tilde{\varphi}(2)^{\prime} d s .
\end{aligned}
$$

Using Lemma 2.2 we obtain

$$
\begin{aligned}
0= & \lambda \int_{0}^{L} \tilde{\psi}^{2}\left(x_{1} \nu_{2}-x_{2} \nu_{1}\right)\left(\nu_{1}+i \nu_{2}\right)^{2} d s+\mu^{2} \int_{0}^{L}\left(x_{1} \nu_{2}-x_{2} \nu_{1}\right)\left(\nu_{1}+i \nu_{2}\right)^{2} d s \\
& +2 i \mu \int_{0}^{L}\left(\kappa \tilde{\psi}(x \cdot \nu)-\tilde{\psi}^{\prime}\left(x_{1} \nu_{2}-x_{2} \nu_{1}\right)\right)\left(\nu_{1}+i \nu_{2}\right)^{2} d s-2 i \int_{0}^{L} \tilde{\psi}(x \cdot \nu \\
& \left.+i\left(x_{1} \nu_{2}-x_{2} \nu_{1}\right)\right)\left(2 i \kappa\left(\tilde{\psi}^{\prime}+i \kappa \tilde{\psi}\right)+\left(\tilde{\psi}^{\prime}+i \kappa \tilde{\psi}\right)^{\prime}\right)\left(\nu_{1}+i \nu_{2}\right)^{2} d s .
\end{aligned}
$$

Since

$$
\begin{aligned}
& \int_{0}^{L} \tilde{\psi}^{\prime}\left(x_{1} \nu_{2}-x_{2} \nu_{1}\right)\left(\nu_{1}+i \nu_{2}\right)^{2} d s \\
& \left.=-\int_{0}^{L} \tilde{\psi}\left(\kappa\left(x \cdot \nu+2 i\left(x_{1} \nu_{2}-x_{2} \nu_{1}\right)\right)\right)-1\right)\left(\nu_{1}+i \nu_{2}\right)^{2}
\end{aligned}
$$

we obtain

$$
A=2 i \int_{0}^{L} \tilde{\psi}\left(2 \kappa\left(x \cdot \nu+i\left(x_{1} \nu_{2}-x_{2} \nu_{1}\right)\right)-1\right)\left(\nu_{1}+i \nu_{2}\right)^{2} d s
$$

3) Take $w=x_{1} \varphi(k)_{2}-x_{2} \varphi(k)_{1}$ in Lemma 2.4. Using (2.4) and integrating we get

$$
\begin{aligned}
0= & \mu \int_{0}^{L}\left(x_{1} \nu_{2}-x_{2} \nu_{1}\right) \tilde{\varphi}(k) d s-\lambda \int_{0}^{L} \tilde{\psi} \tilde{\varphi}(k-1)\left(x_{1} \nu_{2}-x_{2} \nu_{1}\right)\left(\nu_{1}+i \nu_{2}\right) d s \\
& +\int_{0}^{L} \tilde{\psi}\left(x \cdot \nu+i\left(x_{1} \nu_{2}-x_{2} \nu_{1}\right)\right) \tilde{\varphi}(k)^{\prime} d s .
\end{aligned}
$$


Using Lemma 2.3 we obtain

$$
\begin{aligned}
0= & (-1)^{p} \lambda^{p-1}\left[\lambda \int_{0}^{L} \tilde{\psi}^{2}\left(x_{1} \nu_{2}-x_{2} \nu_{1}\right)\left(\nu_{1}+i \nu_{2}\right)^{k} d s+\mu^{2} \int_{0}^{L}\left(x_{1} \nu_{2}-x_{2} \nu_{1}\right)\right. \\
& \left.\times\left(\nu_{1}+i \nu_{2}\right)^{k} d s\right]-\mu \sum_{j=1}^{p-1} \lambda^{p-j} \int_{0}^{L} a_{j, k-1} \tilde{\psi}\left(x_{1} \nu_{2}-x_{2} \nu_{1}\right) \\
& \times\left(\nu_{1}+i \nu_{2}\right)^{k} d s-\sum_{j=1}^{p-1} \lambda^{p-j} \int_{0}^{L} b_{j, k-1} \tilde{\psi}\left(x_{1} \nu_{2}-x_{2} \nu_{1}\right)\left(\nu_{1}+i \nu_{2}\right)^{k} d s \\
& +\mu^{2} \sum_{j=2}^{p} \lambda^{p-j} \int_{0}^{L} c_{j, k}\left(x_{1} \nu_{2}-x_{2} \nu_{1}\right)\left(\nu_{1}+i \nu_{2}\right)^{k} d s \\
& +\mu \sum_{j=1}^{p} \lambda^{p-j} \int_{0}^{L} d_{j, k}\left(x_{1} \nu_{2}-x_{2} \nu_{1}\right)\left(\nu_{1}+i \nu_{2}\right)^{k} d s \\
& +\mu \sum_{j=2}^{p} \lambda^{p-j} \int_{0}^{L}\left(c_{j, k}^{\prime}+i k \kappa c_{j, k}\right) \tilde{\psi}\left(x \cdot \nu+i\left(x_{1} \nu_{2}-x_{2} \nu_{1}\right)\right)\left(\nu_{1}+i \nu_{2}\right)^{k} d s \\
& +\sum_{j=1}^{p} \lambda^{p-j} \int_{0}^{L}\left(d_{j, k}^{\prime}+i k \kappa d_{j, k}\right) \tilde{\psi}\left(x \cdot \nu+i\left(x_{1} \nu_{2}-x_{2} \nu_{1}\right)\right)\left(\nu_{1}+i \nu_{2}\right)^{k} d s \\
& +(-1)^{p} \lambda^{p-1} \mu i k \int_{0}^{L} \kappa \tilde{\psi}\left(x \cdot \nu+i\left(x_{1} \nu_{2}-x_{2} \nu_{1}\right)\right)\left(\nu_{1}+i \nu_{2}\right)^{k} d s .
\end{aligned}
$$

The coefficient of $\lambda^{p-1} \mu$ is given by

$$
\int_{0}^{L}\left[\left(d_{1, k}-\left(\left(a_{1, k-1}+(-1)^{p} k \kappa\right)\left(x_{1} \nu_{2}-x_{2} \nu_{1}\right)+(-1)^{p-1} i k \kappa(x \cdot \nu)\right) \tilde{\psi}\right]\left(\nu_{1}+i \nu_{2}\right)^{k} d s\right.
$$

Since

$$
\begin{aligned}
& \int_{0}^{L}\left(\tilde{\psi}^{\prime}+p i \kappa \tilde{\psi}\right)\left(x_{1} \nu_{2}-x_{2} \nu_{1}\right)\left(\nu_{1}+i \nu_{2}\right)^{k} d s=\int_{0}^{L}\left(x_{1} \nu_{2}-x_{2} \nu_{1}\right)\left(\nu_{1}+i \nu_{2}\right)^{p} \\
& \times\left(\tilde{\psi}\left(\nu_{1}+i \nu_{2}\right)^{p}\right)^{\prime} d s=-\int_{0}^{L}\left(\kappa\left(x \cdot \nu+i p\left(x_{1} \nu_{2}-x_{2} \nu_{1}\right)\right)-1\right) \tilde{\psi}\left(\nu_{1}+i \nu_{2}\right)^{k} d s
\end{aligned}
$$

we obtain

$$
B_{1, k}=(-1)^{p} k i \int_{0}^{L} \tilde{\psi}\left(\kappa\left(2 x \cdot \nu+i k\left(x_{1} \nu_{2}-x_{2} \nu_{1}\right)\right)-1\right)\left(\nu_{1}+i \nu_{2}\right)^{k} d s .
$$

The proof of the Lemma is complete. 


\section{Lemma 2.6.}

1) We have

$$
\int_{\partial \Omega} \psi^{2}\left(n_{1}+i n_{2}\right) d \sigma=0
$$

2) We have

$$
\lambda \int_{0}^{L} \tilde{\psi}^{2}\left(\nu_{1}+i \nu_{2}\right)^{3} d s+\mu^{2} \int_{0}^{L}\left(\nu_{1}+i \nu_{2}\right)^{3} d s+a \mu+b=0,
$$

where $a$ and $b$ are independent of $\lambda$ and $\mu$. Moreover

$$
a=-8 \int_{0}^{L} \kappa \tilde{\psi}\left(\nu_{1}+i \nu_{2}\right)^{3} d s \quad \text { and } \quad b=-4 \int_{0}^{L}\left(\tilde{\psi}^{\prime}+i \kappa \tilde{\psi}\right)^{2}\left(\nu_{1}+i \nu_{2}\right)^{3} d s .
$$

3) Let $k=2 p+1$ with $p \geq 2$. We have

$$
\begin{aligned}
0= & (-1)^{p} \lambda^{p-1}\left[\lambda \int_{0}^{L} \tilde{\psi}^{2}\left(\nu_{1}+i \nu_{2}\right)^{k} d s+\mu^{2} \int_{0}^{L}\left(\nu_{1}+i \nu_{2}\right)^{k} d s\right] \\
& +\mu^{2} \sum_{j=2}^{p} \alpha_{j, k} \lambda^{p-j}+\mu \sum_{j=1}^{p} \beta_{j, k} \lambda^{p-j}+\sum_{j=1}^{p} \gamma_{j, k} \lambda^{p-j},
\end{aligned}
$$

where $\alpha_{j, k}, \beta_{j, k}$ and $\gamma_{j, k}$ are independent of $\lambda$ and $\mu$. Moreover

$$
\begin{aligned}
& \alpha_{2, k}=(-1)^{p} \frac{2}{3}(p-1) p(p+1)(p+2) \int_{0}^{L} \kappa^{2}\left(\nu_{1}+i \nu_{2}\right)^{k} d s \\
& \beta_{1, k}=(-1)^{p-1}\left(k^{2}-1\right) \int_{0}^{L} \kappa \tilde{\psi}\left(\nu_{1}+i \nu_{2}\right)^{k} d s
\end{aligned}
$$

and

$$
\begin{aligned}
\gamma_{1, k}= & (-1)^{p-1} \frac{2 p(p+1)}{3} \int_{0}^{L}\left(3 \tilde{\psi}^{2}+2(2 p+1) i \kappa \tilde{\psi} \tilde{\psi}^{\prime}\right. \\
& \left.-\left(p^{2}+p+1\right) \kappa^{2} \tilde{\psi}^{2}\right)\left(\nu_{1}+i \nu_{2}\right)^{k} d s
\end{aligned}
$$

Proof. 1) Taking $w=\varphi(1)$ in Lemma 2.4 and integrating, we get

$$
\int_{\partial \Omega} \psi^{2}\left(n_{1}+i n_{2}\right) d s=0
$$

2) Take $w=\varphi(3)$ in Lemma 2.4. Using Lemmas 2.1 and 2.2 and integrating we get

$$
\mu \int_{0}^{L}\left(\nu_{1}+i \nu_{2}\right) \tilde{\varphi}(2) d s-\lambda \int_{0}^{L} \tilde{\psi}^{2}\left(\nu_{1}+i \nu_{2}\right)^{3} d s+2 i \int_{0}^{L} \tilde{\psi}\left(\nu_{1}+i \nu_{2}\right) \tilde{\varphi}(2)^{\prime} d s=0 .
$$


Using Lemma 2.2 again we obtain

$$
\begin{aligned}
0= & \lambda \int_{0}^{L} \tilde{\psi}^{2}\left(\nu_{1}+i \nu_{2}\right)^{3} d s+\mu^{2} \int_{0}^{L}\left(\nu_{1}+i \nu_{2}\right)^{3} d s-2 \mu \int_{0}^{L}\left(\kappa \tilde{\psi}+i \tilde{\psi}^{\prime}\right) \\
& \times\left(\nu_{1}+i \nu_{2}\right)^{3} d s+4 \int_{0}^{L} \tilde{\psi}\left(2 i \kappa\left(\tilde{\psi}^{\prime}+i \kappa \tilde{\psi}\right)+\left(\tilde{\psi}^{\prime}+i \kappa \tilde{\psi}\right)^{\prime}\right)\left(\nu_{1}+i \nu_{2}\right)^{3} d s .
\end{aligned}
$$

Since

$$
\int_{0}^{L} \tilde{\psi}^{\prime}\left(\nu_{1}+i \nu_{2}\right)^{3} d s=-3 i \int_{0}^{L} \kappa \tilde{\psi}\left(\nu_{1}+i \nu_{2}\right)^{3} d s
$$

we obtain

$$
a=-8 \int_{0}^{L} \kappa \tilde{\psi}\left(\nu_{1}+i \nu_{2}\right)^{3} d s
$$

Now integrating by parts we have

$$
\int_{0}^{L}\left(\tilde{\psi}^{\prime}+i \kappa \tilde{\psi}\right)^{\prime} \tilde{\psi}\left(\nu_{1}+i \nu_{2}\right)^{3} d s=-\int_{0}^{L}\left(\tilde{\psi}^{\prime}+3 i \kappa \tilde{\psi}\right)\left(\tilde{\psi}^{\prime}+i \kappa \tilde{\psi}\right)\left(\nu_{1}+i \nu_{2}\right)^{3} d s
$$

and we obtain $b$.

3) Take $w=\varphi(k)$ in Lemma 2.4. Using Lemmas 2.1 and 2.2 and integrating we get

$$
\begin{aligned}
0= & \mu \int_{0}^{L} \tilde{\varphi}(k-1)\left(\nu_{1}+i \nu_{2}\right) d s-\lambda \int_{0}^{L} \tilde{\psi} \tilde{\varphi}(k-2)\left(\nu_{1}+i \nu_{2}\right)^{2} d s \\
& +2 i \int_{0}^{L} \tilde{\psi}\left(\nu_{1}+i \nu_{2}\right) \tilde{\varphi}(k-1)^{\prime} d s .
\end{aligned}
$$

Using Lemma 2.3 we obtain

$$
\begin{aligned}
0= & (-1)^{p} \lambda^{p-1}\left[\lambda \int_{0}^{L} \tilde{\psi}^{2}\left(\nu_{1}+i \nu_{2}\right)^{k} d s+\mu^{2} \int_{0}^{L}\left(\nu_{1}+i \nu_{2}\right)^{k} d s\right] \\
& -\mu \sum_{j=1}^{p-1} \lambda^{p-j} \int_{0}^{L} a_{j, k-2} \tilde{\psi}\left(\nu_{1}+i \nu_{2}\right)^{k} d s-\sum_{j=1}^{p-1} \lambda^{p-j} \int_{0}^{L} b_{j, k-2} \\
& \times \tilde{\psi}\left(\nu_{1}+i \nu_{2}\right)^{k} d s+\mu^{2} \sum_{j=2}^{p} \lambda^{p-j} \int_{0}^{L} c_{j, k-1}\left(\nu_{1}+i \nu_{2}\right)^{k} d s+\mu \sum_{j=1}^{p} \lambda^{p-j} \\
& \times \int_{0}^{L} d_{j, k-1}\left(\nu_{1}+i \nu_{2}\right)^{k} d s+\mu \sum_{j=2}^{p} \lambda^{p-j} \int_{0}^{L} 2 i\left(c_{j, k-1}^{\prime}+i(k-1) \kappa c_{j, k-1}\right) \\
& \times \tilde{\psi}\left(\nu_{1}+i \nu_{2}\right)^{k} d s+\sum_{j=1}^{p} \lambda^{p-j} \int_{0}^{L} 2 i\left(d_{j, k-1}^{\prime}+i(k-1) \kappa d_{j, k-1}\right) \\
& \times \tilde{\psi}\left(\nu_{1}+i \nu_{2}\right)^{k} d s+2(-1)^{p-1} \lambda^{p-1} \mu(k-1) \int_{0}^{L} \kappa \tilde{\psi}\left(\nu_{1}+i \nu_{2}\right)^{k} d s
\end{aligned}
$$


We have

$$
\alpha_{2, k}=\int_{0}^{L} c_{2,2 p}\left(\nu_{1}+i \nu_{2}\right)^{k} d s .
$$

Using Lemma 2.3 3b) we obtain

$$
\alpha_{2, k}=2 i \sum_{j=1}^{p-1}(-1)^{p-j-1} \int_{0}^{L}\left(a_{1,2 j+1}^{\prime}+(2 j+1) i \kappa a_{1,2 j+1}\right)\left(\nu_{1}+i \nu_{2}\right)^{k} d s .
$$

For $j=1, \ldots, p-1$ we write

$$
\begin{aligned}
& \int_{0}^{L}\left(a_{1,2 j+1}^{\prime}+(2 j+1) i \kappa a_{1,2 j+1}\right)\left(\nu_{1}+i \nu_{2}\right)^{k} d s=\int_{0}^{L}\left(a_{1,2 j+1}\left(\nu_{1}+i \nu_{2}\right)^{2 j+1}\right)^{\prime} \\
& \quad \times\left(\nu_{1}+i \nu_{2}\right)^{k-2 j-1} d s=-2 i(p-j) \int_{0}^{L} a_{1,2 j+1} \kappa\left(\nu_{1}+i \nu_{2}\right)^{k} d s,
\end{aligned}
$$

hence using Lemma 2.31 )

$$
\begin{aligned}
\alpha_{2, k} & =4 \sum_{j=1}^{p-1}(-1)^{p-j-1}(p-j) \int_{0}^{L} a_{1,2 j+1} \kappa\left(\nu_{1}+i \nu_{2}\right)^{k} d s \\
& =8 \sum_{j=1}^{p-1}(-1)^{p} j(j+1)(p-j) \int_{0}^{L} \kappa^{2}\left(\nu_{1}+i \nu_{2}\right)^{k} d s \\
& =(-1)^{p} \frac{2}{3}(p-1) p(p+1)(p+2) \int_{0}^{L} \kappa^{2}\left(\nu_{1}+i \nu_{2}\right)^{k} d s .
\end{aligned}
$$

Now the coefficient of $\lambda^{p-1} \mu$ is given by

$$
\int_{0}^{L}\left(\left(2(-1)^{p-1}(k-1) \kappa-a_{1, k-2}\right) \tilde{\psi}+d_{1, k-1}\right)\left(\nu_{1}+i \nu_{2}\right)^{k} d s .
$$

Since

$$
\begin{aligned}
\int_{0}^{L}\left(\tilde{\psi}^{\prime}+p i \kappa \tilde{\psi}\right)\left(\nu_{1}+i \nu_{2}\right)^{k} d s & =\int_{0}^{L}\left(\nu_{1}+i \nu_{2}\right)^{p+1}\left(\tilde{\psi}\left(\nu_{1}+i \nu_{2}\right)^{p}\right)^{\prime} d s \\
& =-(p+1) i \int_{0}^{L} \kappa \tilde{\psi}\left(\nu_{1}+i \nu_{2}\right)^{k} d s
\end{aligned}
$$

we obtain

$$
\beta_{1, k}=(-1)^{p-1}\left(k^{2}-1\right) \int_{0}^{L} \kappa \tilde{\psi}\left(\nu_{1}+i \nu_{2}\right)^{k} d s .
$$

Finally from the proof above we have

$\gamma_{1, k}=2 i \int_{0}^{L}\left(d_{1, k-1}^{\prime}+i(k-1) \kappa d_{1, k-1}\right) \tilde{\psi}\left(\nu_{1}+i \nu_{2}\right)^{k} d s-\int_{0}^{L} b_{1, k-2} \tilde{\psi}\left(\nu_{1}+i \nu_{2}\right)^{k} d s$ 
Using Lemma 2.3 3a) we obtain

$$
\gamma_{1, k}=2 i \sum_{j=1}^{p}(-1)^{p-j} \int_{0}^{L}\left(d_{1,2 j}^{\prime}+2 i j \kappa d_{1,2 j}\right) \tilde{\psi}\left(\nu_{1}+i \nu_{2}\right)^{k} d s .
$$

For $j=1, \ldots, p$ we write

$$
\begin{aligned}
& \int_{0}^{L}\left(d_{1,2 j}^{\prime}+2 i j \kappa d_{1,2 j}\right) \tilde{\psi}\left(\nu_{1}+i \nu_{2}\right)^{k} d s=\int_{0}^{L}\left(d_{1,2 j}\left(\nu_{1}+i \nu_{2}\right)^{2 j}\right)^{\prime} \\
& \quad \times \tilde{\psi}\left(\nu_{1}+i \nu_{2}\right)^{k-2 j} d s=-\int_{0}^{L} d_{1,2 j}\left(\tilde{\psi}^{\prime}+(k-2 j) i \kappa \tilde{\psi}\left(\nu_{1}+i \nu_{2}\right)^{k} d s\right.
\end{aligned}
$$

hence

$$
\gamma_{1, k}=2 i \sum_{j=1}^{p}(-1)^{p-j+1} \int_{0}^{L}\left(\tilde{\psi}^{\prime}+(k-2 j) i \kappa \tilde{\psi}\right) d_{1,2 j}\left(\nu_{1}+i \nu_{2}\right)^{k} d s
$$

and using Lemma 2.32 ) the result easily follows.

The proof of the Lemma is complete.

Lemma 2.7. $\quad$ Assume that condition ii) in Definition 1.1 holds. Given any $\psi \in C^{\infty}(\partial \Omega)$, and given any $\lambda \in \mathbb{R}$ there exist at most one $\mu \in \mathbb{R}$ such that the Cauchy problem

$$
\Delta v=-\lambda v-\mu \quad \text { in } \quad \Omega, \quad v=0 \quad \text { and } \quad \frac{\partial v}{\partial n}=\psi \quad \text { on } \quad \partial \Omega
$$

has a solution.

Proof. Assume that there exists a $\lambda \in \mathbb{R}$ for which (2.5) has a solution for two different values $\mu_{1}$ and $\mu_{2}$. Denote by $v_{1}$ and $v_{2}$ two solutions corresponding to $\mu_{1}$ and $\mu_{2}$ respectively. The function $z=v_{1}-v_{2}$ satisfies

$$
\Delta z=-\lambda z+\mu_{2}-\mu_{1} \quad \text { in } \quad \Omega, \quad z=0 \quad \text { and } \quad \frac{\partial z}{\partial n}=0 \quad \text { on } \quad \partial \Omega .
$$

Let $\mu=\mu_{1}-\mu_{2}$ and $\psi \equiv 0$ in Lemma 2.6 2). We obtain

$$
\left(\mu_{1}-\mu_{2}\right)^{2} \int_{0}^{L}\left(\nu_{1}+i \nu_{2}\right)^{3} d s=0
$$

and we arrive at a contradiction.

The next lemma is an immediate consequence of Lemma 2.6 2). 
Lemma 2.8. Assume that condition ii) in Definition 1.1 holds. Given any $\psi \in C^{\infty}(\partial \Omega)$ satisfying $d_{3}\left(\psi^{2}\right) \neq 0$, and given any $\mu \in \mathbb{R}$ there exist at most one $\lambda \in \mathbb{R}$ such that the Cauchy problem (2.5) has a solution.

Lemma 2.9. Assume that condition iii) in Definition 1.3 holds. Given any $\psi \in C^{\infty}(\partial \Omega)$, and given any $\lambda \in \mathbb{R}$ there exist at most one $\mu \in \mathbb{R}$ such that the Cauchy problem (2.5) has a solution.

Proof. Assume that there exists a $\lambda \in \mathbb{R}$ for which (2.5) has a solution for two different values $\mu_{1}$ and $\mu_{2}$. Denote by $v_{1}$ and $v_{2}$ two solutions corresponding to $\mu_{1}$ and $\mu_{2}$ respectively. The function $z=v_{1}-v_{2}$ satisfies

$$
\Delta z=-\lambda z+\mu_{2}-\mu_{1} \quad \text { in } \quad \Omega, \quad z=0 \quad \text { and } \quad \frac{\partial z}{\partial n}=0 \quad \text { on } \quad \partial \Omega .
$$

Let $\mu=\mu_{1}-\mu_{2}$ and $\psi \equiv 0$ in Lemma 2.52 ). We obtain

$$
\left(\mu_{1}-\mu_{2}\right)^{2} \int_{0}^{L}\left(\nu_{1}+i \nu_{2}\right)^{2}\left(x_{1} \nu_{2}-x_{2} \nu_{1}\right) d s=0
$$

and we arrive at a contradiction.

The next lemma is an immediate consequence of Lemma 2.52 ).

Lemma 2.10. Assume that condition iii) in Definition 1.3 holds. Given any $\psi \in C^{\infty}(\partial \Omega)$ satisfying $d_{2}\left(\psi^{2} l\right) \neq 0$, and given any $\mu \in \mathbb{R}$ there exist at most one $\lambda \in \mathbb{R}$ such that the Cauchy problem (2.5) has a solution.

\section{§3. Proof of Theorem 1.1 and Corollary 1.1}

Assume first that $0 \in \Omega$. Since $\partial \Omega$ has positive curvature, the curve $\partial \Omega$ turns continuously. To each point $x=x(s) \in \partial \Omega$ we can associate a unique $\theta$ (modulo $2 \pi$ ) and $\theta$ makes a complete circuit $0 \leq \theta \leq 2 \pi$ as $0 \leq s \leq L$. For each angle $\theta, 0 \leq \theta<2 \pi$, let $h(\theta)$ denote the distance from the origin to the supporting line of $\Omega$ with outward normal $\nu=(\cos \theta, \sin \theta)$. We have

$$
h(\theta)=x \cdot \nu
$$

and $h$ has period $2 \pi$. From the Serret-Frenet formulas we can derive the following second order ordinary differential equation involving the support function $h$ and the radius of curvature $\rho$ :

$$
h(\theta)+h^{\prime \prime}(\theta)=\rho(\theta) .
$$


When $0 \notin \Omega$, the support function is defined in the following way. By translation there exists $a=\left(a_{1}, a_{2}\right) \in \mathbb{R}^{2}$ such that $0 \in \tilde{\Omega}=a+\Omega$. If $\tilde{h}$ denotes the support function of $\tilde{\Omega}$ we have

$$
h(\theta)=-a_{1} \cos \theta-a_{2} \sin \theta+\tilde{h}(\theta) .
$$

We refer the reader to Flanders [7] and the references therein for a detailed discussion.

For any $f:[0,2 \pi] \rightarrow \mathbb{C}$ such that $f \in L^{2}[0,2 \pi]$ we denote by

$$
c_{n}(f)=\frac{1}{2 \pi} \int_{0}^{2 \pi} f(\theta) e^{-i n \theta} d \theta
$$

$n \in \mathbb{Z}$, the Fourier coefficients.

We first show that when $\Omega \subset \mathbb{R}^{2}$ is of class $\mathcal{B}$, then $\Omega$ is of constant width.

Proposition 3.1. Let $\Omega \subset \mathbb{R}^{2}$ be a bounded open set with $C^{2}$ boundary and positive curvature. Assume that condition iii) in Definition 1.1 holds. Then $\Omega$ has constant width.

Proof. We have $d_{2 n}(1)=\overline{c_{2 n}(\rho)}=0$ for $n \in \mathbb{Z}^{*}$. Since

$$
c_{n}(\rho)=c_{n}\left(h+h^{\prime \prime}\right)=\left(1-n^{2}\right) c_{n}(h) \quad \forall n \in \mathbb{Z},
$$

we deduce that $c_{2 n}(h)=0$ for $n \neq 0$, hence

$$
h(\theta)+h(\theta+\pi)=2 c_{0}(h) \quad \forall \theta \in[0,2 \pi]
$$

and this means that $\Omega$ is of constant width.

Proof of Theorem 1.1. Let $(\lambda, \mu) \in \mathbb{R}^{2}$ be such that (1.1)-(1.2) has a solution $u \in C^{\infty}(\bar{\Omega})$ satisfying

$$
\frac{\partial u}{\partial n}=\psi \quad \text { on } \partial \Omega
$$

for some $\psi \in C^{\infty}(\partial \Omega)$ satisfying the conditions of Theorem 1.1.

We shall need the following lemma.

Lemma 3.1. $\quad d_{2(2 m+1)}(l) \neq 0, d_{2 n+1}(1)=0$ for $n \geq m+1$ and $d_{2 n}(l)=0$ for $n \geq 2(m+1)$. 
Proof. We have seen in the proof of Proposition 3.1 that $c_{2 n}(h)=0$ for $n \in \mathbb{Z}^{*}$. In the same way we can show that $c_{2 n+1}(h)=0$ for $n \geq m+1$ and $c_{2 m+1}(h) \neq 0$. Therefore we can write

$$
h(\theta)=c_{0}(h)+\sum_{n=-m-1}^{m} c_{2 n+1}(h) e^{(2 n+1) i \theta}, \quad 0 \leq \theta<2 \pi .
$$

We have $d_{n}(l)=-\overline{c_{n}\left(h^{\prime} \rho\right)}$ with $h^{\prime}=-l \circ x \circ s$. Since

$$
\begin{aligned}
c_{n}\left(h^{\prime} \rho\right) & =\sum_{j=-\infty}^{+\infty} c_{n-j}\left(h^{\prime}\right) c_{j}(\rho) \\
& =c_{n}\left(h^{\prime}\right) c_{0}(\rho)+\sum_{j=-m-1}^{m} c_{n-2 j-1}\left(h^{\prime}\right) c_{2 j+1}(\rho),
\end{aligned}
$$

the lemma follows easily.

Assume first that there exists $n \in \mathbb{Z}$ such that

$$
d_{2 n+1}(1) d_{3}\left(\psi^{2}\right) \neq d_{2 n+1}\left(\psi^{2}\right) d_{3}(1) .
$$

Then we have

$$
c_{2 n+1}(\rho) c_{3}\left(\Psi^{2} \rho\right) \neq c_{3}(\rho) c_{2 n+1}\left(\Psi^{2} \rho\right),
$$

for some $n \in \mathbb{Z}$, where

$$
\Psi(\theta)=\psi(x(s(\theta)))=\tilde{\psi}(s(\theta)) .
$$

Since $c_{1}(\rho)=c_{-1}(\rho)=0$, Lemma 2.61$)$ implies that $n \in \mathbb{Z} \backslash\{-1,0\}$. Then $k_{1}(\psi)<\infty$. If $c_{3}\left(\Psi^{2} \rho\right)=0$, Lemma 2.62$)$ implies that $\mu$ satisfies a polynomial equation of degree 2 . This equation has at most two real roots $\mu_{1}$ and $\mu_{2}$. (3.1) and Lemma 2.63 ) with $\mu=\mu_{j}, j=1,2$, imply that $\lambda$ satisfies two polynomial equations of degree $k_{1}(\psi) / 2$. Therefore there exist at most $k_{1}(\psi)$ different $\lambda_{k}$. Using Lemma 2.7 we conclude that there exist at most $k_{1}(\psi)$ different pairs of coefficients $\left(\lambda_{k}, \mu_{k}\right) \in \mathbb{R}^{2}$ such that the Cauchy problem (1.5) has a solution. Now, if $c_{3}\left(\Psi^{2} \rho\right) \neq 0$, Lemma 2.62$)$ implies that $\lambda$ is a polynomial of degree 2 in $\mu$. Using (3.1) and Lemma 2.63 ) we deduce that $\mu$ satisfies a polynomial equation of degree $k_{1}(\psi)$. Using Lemma 2.8 we conclude that there exist at most $k_{1}(\psi)$ different pairs of coefficients $\left(\lambda_{k}, \mu_{k}\right) \in \mathbb{R}^{2}$ such that the Cauchy problem (1.5) has a solution.

Now suppose that (3.1) does not hold. Then we have

$$
c_{2 n+1}\left(\Psi^{2} \rho\right)=\alpha c_{2 n+1}(\rho) \quad \forall n \in \mathbb{Z},
$$


with

$$
\alpha=\frac{c_{3}\left(\Psi^{2} \rho\right)}{c_{3}(\rho)} .
$$

Since $c_{2 j}\left(h^{\prime}\right)=0$ for $j \in \mathbb{Z}$, using (3.2) we deduce that

$$
\begin{aligned}
c_{2 n}\left(\Psi^{2} h^{\prime} \rho\right) & =\sum_{j=-\infty}^{\infty} c_{2(n-j)-1}\left(\Psi^{2} \rho\right) c_{2 j+1}\left(h^{\prime}\right) \\
& =\alpha \sum_{j=-\infty}^{\infty} c_{2(n-j)-1}(\rho) c_{2 j+1}\left(h^{\prime}\right)=\alpha c_{2 n}\left(h^{\prime} \rho\right),
\end{aligned}
$$

for $n \in \mathbb{Z}$.

Suppose that $c_{3}\left(\Psi^{2} \rho\right)=0$. Then $\alpha=0$ and (3.2) implies that

$$
c_{2 n+1}\left(\Psi^{2} \rho\right)=0 \quad \forall n \in \mathbb{Z} .
$$

We have

$$
c_{2 n+1}\left(\Psi^{2} \rho\right)=c_{2 n+1}\left(\Psi^{2}\right) c_{0}(\rho)+\sum_{j=-m-1}^{m} c_{2(n-j)}\left(\Psi^{2}\right) c_{2 j+1}(\rho),
$$

for $n \in \mathbb{Z}$. We can write $\Psi^{2}=A+B$ with

$$
A(\theta)=\sum_{n=-\infty}^{\infty} c_{2 n}\left(\Psi^{2}\right) e^{2 i n \theta} \text { and } B(\theta)=\sum_{n=-\infty}^{\infty} c_{2 n+1}\left(\Psi^{2}\right) e^{(2 n+1) i \theta},
$$

for $\theta \in[0,2 \pi] .(3.4)$ and (3.5) imply that

$$
\begin{aligned}
B(\theta) & =-\frac{1}{c_{0}(\rho)} \sum_{j=-m-1}^{m} c_{2 j+1}(\rho) e^{(2 j+1) i \theta}\left(\sum_{n=-\infty}^{\infty} c_{2(n-j)}\left(\Psi^{2}\right) e^{2(n-j) i \theta}\right) \\
& =\frac{1}{c_{0}(\rho)} A(\theta)\left(c_{0}(\rho)-\rho(\theta)\right)
\end{aligned}
$$

for $\theta \in[0,2 \pi]$. Therefore

$$
\Psi^{2}(\theta)=\frac{1}{c_{0}(\rho)} A(\theta) \rho(\theta+\pi), \quad 0 \leq \theta<2 \pi,
$$

from which we deduce that

$$
\Psi^{2}(\theta) \rho(\theta)=\Psi^{2}(\theta+\pi) \rho(\theta+\pi), \quad 0 \leq \theta<2 \pi,
$$

and we reach a contradiction with $(*)$. Therefore $c_{3}\left(\Psi^{2} \rho\right) \neq 0$. 
We claim that $k_{2, m}(\psi)<\infty$ or $k_{3, m}(\psi)<\infty$. Indeed suppose the contrary. Since $\psi$ is not identically constant, there exists $p \in\{1, \ldots, 2(2 m+1)\}$ such that

$$
\Psi(\theta)=\sum_{n=-p}^{p} c_{n}(\Psi) e^{i n \theta}, \quad 0 \leq \theta<2 \pi
$$

with $c_{p}(\Psi) \neq 0$. We have $c_{2(m+p)+1}\left(\Psi^{2} \rho\right)=c_{p}(\Psi)^{2} c_{2 m+1}(\rho) \neq 0$. From Lemma 3.1 and (3.2) we deduce that $c_{2 n+1}\left(\Psi^{2} \rho\right)=0$ for $n \geq m+1$. Then we get a contradiction, and our claim is proved.

Since $c_{3}\left(\Psi^{2} \rho\right) \neq 0$, Lemma 2.62$)$ implies that $\lambda$ is a polynomial of degree 2 in $\mu$. Suppose first that $k_{2, m}(\psi)<\infty$. Since $c_{2 n+1}\left(\Psi^{2} \rho\right)=0$ for $n \geq m+1$, Lemma 2.63$)$ implies that $\mu$ satisfies a polynomial equation of degree $k_{2, m}(\psi)$. Using Lemma 2.8 we conclude that there exist at most $k_{2, m}(\psi)$ different pairs of coefficients $\left(\lambda_{k}, \mu_{k}\right) \in \mathbb{R}^{2}$ such that the Cauchy problem (1.5) has a solution. Now assume that $k_{2, m}(\psi)=\infty$. Then $k_{3, m}(\psi)<+\infty$. We can write

$$
c_{2 n}\left(\Psi\left(h-h^{\prime \prime}+2 i n h^{\prime}\right)\right)=c_{2 n}(\Psi) c_{0}(h)+\sum_{j=-m-1}^{m} \omega_{j, n} c_{2(n-j)-1}(\Psi) c_{2 j+1}(h),
$$

where $\omega_{j, n}=2\left(2 j^{2}+(1-n)(2 j+1)\right)$. If $n \geq 2(m+1)$ we obtain

$$
c_{2 n}\left(\Psi\left(h-h^{\prime \prime}+2 i n h^{\prime}\right)\right)=c_{2 n}(\Psi) c_{0}(h),
$$

and we conclude that there exists $n \geq 2(m+1)$ such that $c_{2 n}\left(\Psi\left(h-h^{\prime \prime}+\right.\right.$ $\left.\left.2 i n h^{\prime}\right)\right) \neq 0$. (3.3) and Lemma 3.1 imply that $c_{2 n}\left(\Psi^{2} h^{\prime} \rho\right)=0$ for $n \geq 2(m+1)$. Then using Lemma 2.53 ) we deduce that $\mu$ satisfies a polynomial equation of degree $k_{3, m}(\psi)$. With the help of Lemma 2.8 we conclude that there exist at most $k_{3, m}(\psi)$ different pairs of coefficients $\left(\lambda_{k}, \mu_{k}\right) \in \mathbb{R}^{2}$ such that the Cauchy problem (1.5) has a solution.

Remark 2. In fact the proof of Theorem 1.1 shows that we can give a better result. Define

$$
\tilde{k}_{2}(\psi)=2 \inf \left\{n \in \mathbb{N}^{*} ; \beta_{1,2 n+1} d_{3}(1) \neq(-1)^{n} a d_{2 n+1}(1)\right\}-1,
$$

and

$$
\tilde{k}_{3}(\psi)=2 \inf \left\{n \in \mathbb{N}^{*} ; B_{1,2 n} d_{3}(1) \neq(-1)^{n} a d_{2 n}(l)\right\}-1 .
$$

If $k_{1}(\psi)=\infty$, then $\tilde{k}_{2}(\psi)<\infty$ or $\tilde{k}_{3}(\psi)<\infty$ and we can take

$$
k_{0}=\min \left(\tilde{k}_{2}(\psi), \tilde{k}_{3}(\psi)\right) .
$$


Moreover, if $c_{3}\left(\psi^{2} \rho\right) \neq 0$, then $k_{1}(\psi)<\infty$ or $\tilde{k}_{2}(\psi)<\infty$ or $\tilde{k}_{3}(\psi)<\infty$ and

$$
k_{0}=\min \left(k_{1}(\psi), \tilde{k}_{2}(\psi), \tilde{k}_{3}(\psi)\right) .
$$

Finally, if $c_{3}\left(\psi^{2} \rho\right)=0$, then $k_{1}(\psi)<\infty$.

Remark 3. Let $\Omega \subset \mathbb{R}^{2}$ be of class $\mathcal{B}$. Let $\psi \in C^{\infty}(\partial \Omega)$ be a real valued function and assume that

$$
\psi=\sum_{j=-p}^{p} c_{j}\left(n_{1}+i n_{2}\right)^{j}
$$

where $p \geq 1, c_{j} \in \mathbb{C}$ for $j \in\{-p, \ldots, p\}$ and $c_{p} \neq 0$. Then there exist at most $k_{1}(\psi)$ different pairs of coefficients $\left(\lambda_{k}, \mu_{k}\right) \in \mathbb{R}^{2}$ such that the Cauchy problem (1.5) has a solution. It is enough to verify that (3.1) holds. Indeed we have $d_{2(m+p)+1}\left(\psi^{2}\right)=\overline{c_{p}^{2}} \overline{c_{2 m+1}(\rho)} \neq 0$ and by Lemma $3.1 d_{2 n+1}(1)=0$ for $n \geq m+1$. Notice that condition $(*)$ is not needed, but it is satisfied. Indeed, let $\phi(\theta)=\Psi^{2}(\theta+\pi) \rho(\theta+\pi)$ for $\theta \in[0,2 \pi]$. We have $c_{2(m+p)+1}\left(\Psi^{2} \rho\right)=$ $c_{p}^{2} c_{2 m+1}(\rho) \neq 0$ and $c_{2(m+p)+1}(\phi)=-c_{p}^{2} c_{2 m+1}(\rho)$.

Proof of Corollary 1.1. By Theorem 1.1 it is enough to show that if $(\lambda, \mu) \in$ $\mathbb{R}^{2}$ and if $v \in C^{\infty}(\bar{\Omega})$ is a solution of the following Cauchy problem

$$
\Delta v=-\lambda v-\mu \leq 0 \quad \text { in } \Omega, \quad v=0 \quad \text { and } \frac{\partial v}{\partial n}=\psi \quad \text { on } \partial \Omega,
$$

where $\psi \in C^{\infty}(\partial \Omega)$ satisfies condition $(*)$, then $\psi$ cannot be identically constant. Suppose the contrary. The Maximum Principle implies that $v>0$ in $\Omega$ or $v \equiv 0$ in $\Omega$. Since $\psi$ satisfies condition $(*), \psi \neq \equiv 0$, hence we have $v>0$ in $\Omega$. Then a result of Serrin [10] asserts that $\Omega$ is a disk, which represents an obvious contradiction to ii) in Definition 1.1.

\section{$\S 4$. Proof of Theorem 1.2 and Corollary 1.2}

Proof of Theorem 1.2. Let $(\lambda, \mu) \in \mathbb{R}^{2}$ be such that (1.1)-(1.2) has a solution $u \in C^{\infty}(\bar{\Omega})$ satisfying

$$
\frac{\partial u}{\partial n}=\psi \quad \text { on } \partial \Omega
$$

for some $\psi \in C^{\infty}(\partial \Omega)$, which is not identically constant.

We can write $h=h_{1}+h_{2}$ with

$$
h_{1}(\theta)=\sum_{n=-\infty}^{+\infty} c_{2 n}(h) e^{2 i n \theta} \quad \text { and } \quad h_{2}(\theta)=\sum_{n=-\infty}^{+\infty} c_{2 n+1}(h) e^{(2 n+1) i \theta} .
$$


Assume first that there exists $n \in \mathbb{Z}$ such that

$$
c_{2 n+1}(\rho) c_{3}\left(\Psi^{2} \rho\right) \neq c_{3}(\rho) c_{2 n+1}\left(\Psi^{2} \rho\right) .
$$

Using the same arguments as in the proof of Theorem 1.1 we obtain that $k_{1}(\psi)<\infty$ and we conclude that there exist at most $k_{1}(\psi)$ different pairs of coefficients $\left(\lambda_{k}, \mu_{k}\right) \in \mathbb{R}^{2}$ such that the Cauchy problem (1.5) has a solution.

Now suppose that (4.1) does not hold. Then we have

$$
c_{2 n+1}\left(\Psi^{2} \rho\right)=\alpha c_{2 n+1}(\rho) \quad \forall n \in \mathbb{Z},
$$

with

$$
\alpha=\frac{c_{3}\left(\Psi^{2} \rho\right)}{c_{3}(\rho)} .
$$

We claim that there exists $n \in \mathbb{Z}^{*}$ such that

$$
c_{2 n}\left(\Psi^{2} h^{\prime} \rho\right) \neq \alpha c_{2 n}\left(h^{\prime} \rho\right) .
$$

Indeed suppose the contrary. $c_{0}\left(h^{\prime} \rho\right)=0$ and by Lemma 2.51$) c_{0}\left(\Psi^{2} h^{\prime} \rho\right)=0$, hence

$$
c_{2 n}\left(\Psi^{2} h^{\prime} \rho\right)=\alpha c_{2 n}\left(h^{\prime} \rho\right) \quad \forall n \in \mathbb{Z} .
$$

Using (4.2) and (4.4) we can write

$$
\begin{aligned}
0 & =c_{2 n}\left(\Psi^{2} h^{\prime} \rho\right)-\alpha c_{2 n}\left(h^{\prime} \rho\right) \\
& =\sum_{j=-\infty}^{+\infty}\left(c_{2 n-j}\left(\Psi^{2} \rho\right)-\alpha c_{2 n-j}(\rho)\right) c_{j}\left(h^{\prime}\right) \\
& =\sum_{j=-\infty}^{+\infty}\left(c_{2 n-j}\left(\Psi^{2} \rho\right)-\alpha c_{2 n-j}(\rho)\right) c_{j}\left(h_{1}^{\prime}\right) \\
& =c_{2 n}\left(\Psi^{2} h_{1}^{\prime} \rho\right)-\alpha c_{2 n}\left(h_{1}^{\prime} \rho\right)
\end{aligned}
$$

for $n \in \mathbb{Z}$. Now (4.2) implies that

$$
\begin{aligned}
c_{2 n+1}\left(\Psi^{2} h_{1}^{\prime} \rho\right) & =\sum_{j=-\infty}^{+\infty} c_{2(n-j)+1}\left(\Psi^{2} \rho\right) c_{2 j}\left(h_{1}^{\prime}\right) \\
& =\alpha \sum_{j=-\infty}^{+\infty} c_{2(n-j)+1}(\rho) c_{2 j}\left(h_{1}^{\prime}\right)=\alpha c_{2 n+1}\left(h_{1}^{\prime} \rho\right),
\end{aligned}
$$

for $n \in \mathbb{Z}$. We have thus proved that

$$
c_{n}\left(\Psi^{2} h_{1}^{\prime} \rho\right)=\alpha c_{n}\left(h_{1}^{\prime} \rho\right) \quad \forall n \in \mathbb{Z}
$$


which implies that $\Psi^{2} h_{1}^{\prime} \rho=\alpha h_{1}^{\prime} \rho$. Using iv) in Definition 1.2 and the fact that $\rho$ is positive we deduce that $\Psi$ is identically constant and we reach a contradiction. Thus our claim is proved. Then $k_{4}(\Psi)<\infty$. If $c_{3}\left(\Psi^{2} \rho\right)=0$, Lemma 2.6 2) implies that $\mu$ satisfies a polynomial equation of degree 2 . This equation has at most two real roots $\mu_{1}$ and $\mu_{2}$. Lemma 2.52 ) and 3) with $\mu=\mu_{j}, j=1,2$, imply that $\lambda$ satisfies two polynomial equations of degree $k_{4}(\psi) / 2$. Therefore there exist at most $k_{4}(\psi)$ different $\lambda_{k}$. Using Lemma 2.7 we conclude that there exist at most $k_{4}(\psi)$ different pairs of coefficients $\left(\lambda_{k}, \mu_{k}\right) \in \mathbb{R}^{2}$ such that the Cauchy problem (1.5) has a solution. Now, if $c_{3}\left(\Psi^{2} \rho\right) \neq 0$, then Lemma 2.6 2) implies that $\lambda$ is a polynomial of degree 2 in $\mu$. Using (4.3) and Lemma 2.53 ) we deduce that $\mu$ satisfies a polynomial equation of degree $k_{4}(\psi)$. Using Lemma 2.8 we conclude that there exist at most $k_{4}(\psi)$ different pairs of coefficients $\left(\lambda_{k}, \mu_{k}\right) \in \mathbb{R}^{2}$ such that the Cauchy problem (1.5) has a solution.

Remark 4. Assume that $\left\{n \in \mathbb{Z}^{*} ; d_{2 n}(1) \neq 0\right\} \neq \emptyset$ is finite. Then condition iv) in Definition 1.2 is satisfied.

Proof of Corollary 1.2. By Theorem 1.2 it is enough to show that if $(\lambda, \mu) \in \mathbb{R}^{2}$ and if $v \in C^{\infty}(\bar{\Omega})$ is a solution of the following Cauchy problem

$$
\Delta v=-\lambda v-\mu \leq 0 \quad \text { in } \quad \Omega, \quad v=0 \quad \text { and } \quad \frac{\partial v}{\partial n}=\psi \quad \text { on } \quad \partial \Omega,
$$

where $\psi \in C^{\infty}(\partial \Omega), \psi \neq \equiv 0$, then $\psi$ cannot be identically constant. Suppose the contrary. The Maximum Principle implies that $v>0$ in $\Omega$ or $v \equiv 0$ in $\Omega$. Since $\psi \not \equiv 0$, we have $v>0$ in $\Omega$. Then a result of Serrin [10] asserts that $\Omega$ is a disk, which represents an obvious contradiction to ii) (or iv)) in Definition 1.2.

\section{$\S 5$. Proof of Theorem 1.3 and Corollary 1.3}

Proof of Theorem 1.3. Let $(\lambda, \mu) \in \mathbb{R}^{2}$ be such that (1.1)-(1.2) has a solution $u \in C^{\infty}(\bar{\Omega})$ satisfying

$$
\frac{\partial u}{\partial n}=\psi \quad \text { on } \partial \Omega
$$

for some $\psi \in C^{\infty}(\partial \Omega)$, which is not identically constant.

Assume first that there exists $n \in \mathbb{Z}$ such that

$$
d_{2 n}(l) d_{2}\left(\psi^{2} l\right) \neq d_{2}(l) d_{2 n}\left(\psi^{2} l\right)
$$

Then we have

$$
c_{2 n}\left(h^{\prime} \rho\right) c_{2}\left(\Psi^{2} h^{\prime} \rho\right) \neq c_{2}\left(h^{\prime} \rho\right) c_{2 n}\left(\Psi^{2} h^{\prime} \rho\right)
$$


for some $n \in \mathbb{Z}$. Since $c_{0}\left(h^{\prime} \rho\right)=0$, Lemma 2.51 ) implies that $n \in \mathbb{Z}^{*}$. Then $k_{5}(\psi)<\infty$. If $c_{2}\left(\Psi^{2} h^{\prime} \rho\right)=0$, Lemma 2.52$)$ implies that $\mu$ satisfies a polynomial equation of degree 2 . This equation has at most two real roots $\mu_{1}$ and $\mu_{2}$. (5.1) and Lemma 2.53 ) with $\mu=\mu_{j}, j=1,2$, imply that $\lambda$ satisfies two polynomial equations of degree $k_{5}(\psi) / 2$. Therefore there exist at most $k_{5}(\psi)$ different $\lambda_{k}$. Using Lemma 2.9 we conclude that there exist at most $k_{5}(\psi)$ different pairs of coefficients $\left(\lambda_{k}, \mu_{k}\right) \in \mathbb{R}^{2}$ such that the Cauchy problem (1.5) has a solution. Now, if $c_{2}\left(\Psi^{2} h^{\prime} \rho\right) \neq 0$, Lemma 2.52$)$ implies that $\lambda$ is a polynomial of degree 2 in $\mu$. Using (5.1) and Lemma 2.53 ) we deduce that $\mu$ satisfies a polynomial equation of degree $k_{5}(\psi)$. Using Lemma 2.10 we conclude that there exist at most $k_{5}(\psi)$ different pairs of coefficients $\left(\lambda_{k}, \mu_{k}\right) \in \mathbb{R}^{2}$ such that the Cauchy problem (1.5) has a solution.

Now suppose that (5.1) does not hold. Then we have

$$
c_{2 n}\left(\Psi^{2} h^{\prime} \rho\right)=\beta c_{2 n}\left(h^{\prime} \rho\right) \quad \forall n \in \mathbb{Z},
$$

with

$$
\beta=\frac{c_{2}\left(\Psi^{2} h^{\prime} \rho\right)}{c_{2}\left(h^{\prime} \rho\right)} .
$$

We claim that there exists $n \in \mathbb{Z} \backslash\{-1,0\}$ such that

$$
c_{2 n+1}\left(\Psi^{2} \rho\right) \neq \beta c_{2 n+1}(\rho) .
$$

Indeed suppose the contrary. $c_{1}(\rho)=0$ and by Lemma 2.6 1) $c_{1}\left(\Psi^{2} \rho\right)=0$, hence

$$
c_{2 n+1}\left(\Psi^{2} \rho\right)=\beta c_{2 n+1}(\rho) \quad \forall n \in \mathbb{Z} .
$$

Using (5.2) and (5.4) with the notations introduced in Section 4, and arguing as in the proof of Theorem 1.2 we obtain

$$
c_{n}\left(\Psi^{2} h_{1}^{\prime} \rho\right)=\beta c_{n}\left(h_{1}^{\prime} \rho\right) \quad \forall n \in \mathbb{Z},
$$

and we reach a contradiction in the same way. Thus our claim is proved. Then $k_{6}(\Psi)<\infty$. If $c_{2}\left(\Psi^{2} h^{\prime} \rho\right)=0$, Lemma 2.52$)$ implies that $\mu$ satisfies a polynomial equation of degree 2 . This equation has at most two real roots $\mu_{1}$ and $\mu_{2}$. Lemma 2.6 2) and 3) with $\mu=\mu_{j}, j=1,2$, imply that $\lambda$ satisfies two polynomial equations of degree $k_{6}(\psi) / 2$. Therefore there exist at most $k_{6}(\psi)$ different $\lambda_{k}$. Using Lemma 2.9 we conclude that there exist at most $k_{6}(\psi)$ different pairs of coefficients $\left(\lambda_{k}, \mu_{k}\right) \in \mathbb{R}^{2}$ such that the Cauchy problem (1.5) has a solution. Now, if $c_{2}\left(\Psi^{2} h^{\prime} \rho\right) \neq 0$, then Lemma 2.52$)$ implies that $\lambda$ is a 
polynomial of degree 2 in $\mu$. Using (5.3) and Lemma 2.53 ) we deduce that $\mu$ satisfies a polynomial equation of degree $k_{6}(\psi)$. Using Lemma 2.10 we conclude that there exist at most $k_{6}(\psi)$ different pairs of coefficients $\left(\lambda_{k}, \mu_{k}\right) \in \mathbb{R}^{2}$ such that the Cauchy problem (1.5) has a solution.

Remark 5. Assume that

a) $d_{2 n+1}(1)=0$ for $n \in \mathbb{Z}$;

b) $l$ has at most countably many zeros.

It is clear from the proof that condition ii) in Definition 1.3 can be omitted and then class $\mathcal{D}$ coincides with class $\mathcal{A}$ defined in [6]. Notice that in Definition 1.1 of [6] some conditions are in fact redundant. Indeed we first note that problem (1.1)-(1.2) being invariant under translations we can assume that $c_{1}(h)=0$. Then iv) in Definition 1.1 of [6] reduces to

c) $d_{2 p+1, q}(1)=0$ for $p \in \mathbb{N}$ and $q \in\{0, \ldots, 2 p+1\}$.

Now it is easy to see that c) is equivalent to a).

Proof of Corollary 1.3. The arguments are the same as in the proof of Corollary 1.2, using Theorem 1.3 instead of Theorem 1.2. This time we obtain a contradiction with ii) (or iii)) in Definition 1.3.

\section{§6. A Remark and the Case of a Nonzero Constant}

We will show first that when $\Omega \subset \mathbb{R}^{2}$ is of class $\mathcal{B}, \mathcal{C}$ or $\mathcal{D}$ then $\Omega$ has the Schiffer property (see also [4]-[6]).

Proposition 6.1. Let $\Omega \subset \mathbb{R}^{2}$ be a simply connected $C^{2, \alpha}$ domain, $\alpha \in$ $(0,1]$. If condition ii) in Definition 1.1 (resp. iii) in Definition 1.3) holds, then $\Omega$ has the Schiffer property.

Proof. Let $v \in C^{2, \alpha}(\bar{\Omega})$ be a solution of problem (1.3)-(1.4) with $\mu \neq 0$. By [7, Theorem $\left.1^{\prime}\right] \partial \Omega$ is real analytic. Then $v$ is also analytic. Since $\psi \equiv 0$, using Lemmas 2.52 ) and 2.62 ) we obtain

$$
\mu^{2} \int_{0}^{L}\left(x_{1} \nu_{2}-x_{2} \nu_{1}\right)\left(\nu_{1}+i \nu_{2}\right)^{2} d s=0
$$

and

$$
\mu^{2} \int_{0}^{L}\left(\nu_{1}+i \nu_{2}\right)^{3} d s=0
$$

and we have a contradiction in both cases. 
Now we shall study the case where $\psi$ is a non zero constant. Assume that $\Omega=\left\{x=\left(x_{1}, x_{2}\right) \in \mathbb{R}^{2} ; x_{1}^{2}+x_{2}^{2}<1\right\}$. For any $\lambda>0$ such that $\sqrt{\lambda}$ is not a zero of $J_{1}$, the function

$$
u(x)=\frac{1}{\sqrt{\lambda}} \frac{J_{0}(\sqrt{\lambda}|x|)-J_{0}(\sqrt{\lambda})}{J_{1}(\sqrt{\lambda})} \quad x \in \Omega,
$$

satisfies the overdetermined Cauchy problem

$$
\begin{aligned}
& \Delta u+\lambda u+\mu=0 \quad \text { in } \Omega, \\
& u=0 \text { and } \quad \frac{\partial u}{\partial n}=-1 \quad \text { on } \partial \Omega
\end{aligned}
$$

with the constant $\mu$ given by

$$
\mu=\frac{\sqrt{\lambda} J_{0}(\sqrt{\lambda})}{J_{1}(\sqrt{\lambda})}
$$

We refer the reader to [11] for more details.

Our purpose is to examine what happens when $\Omega$ is not a disk. In the particular case where $\mu=0$, Berenstein ([1, Proposition 3]) has shown that, when $\Omega$ is simply connected and $\Omega$ is not a disk, there exist at most finitely many eigenfunctions for the Dirichlet problem which have constant normal derivative. We give below some partial results in the general case where $\mu \in \mathbb{R}$.

Proposition 6.2. Let $\Omega \subset \mathbb{R}^{2}$ be of class $\mathcal{B}$. Let $\psi$ be a non zero constant. Then

$$
k_{7}=2 \inf \left\{n \geq 2 ; 2 d_{3}\left(K^{2}\right) d_{2 n+1}(1) \neq n(n+1) d_{3}(1) d_{2 n+1}\left(K^{2}\right)\right\}-2
$$

is finite and there exist at most $k_{7}$ different pairs of coefficients $\left(\lambda_{k}, \mu_{k}\right) \in \mathbb{R}^{2}$ such that the Cauchy problem (1.5) has a solution.

Lemma 6.1. For all $n \in \mathbb{N}$, there exists $p \geq n$ such that $d_{2 p+1}\left(K^{2}\right) \neq 0$.

Proof. For all $n \in \mathbb{N}^{*}$ we have

$$
0=c_{2 n}(1)=c_{0}(\rho) c_{2 n}\left(\frac{1}{\rho}\right)+\sum_{j=-m-1}^{m} c_{2(n-j)-1}\left(\frac{1}{\rho}\right) c_{2 j+1}(\rho) .
$$

Assume first that $d_{2 p+1}\left(K^{2}\right)=0$ for $p \in \mathbb{Z}$. Then (6.1) implies that

$$
c_{2 n}\left(\frac{1}{\rho}\right)=0 \quad \forall n \in \mathbb{N}^{*},
$$


and we deduce that $\rho$ is constant, a contradiction with ii) in Definition 1.1. Therefore there exists $p \in \mathbb{N}$ such that $d_{2 p+1}\left(K^{2}\right) \neq 0$. Suppose that the set $\left\{n \in \mathbb{N} ; d_{2 n+1}\left(K^{2}\right) \neq 0\right\}$ is finite and define

$$
r=\max \left\{n \in \mathbb{N} ; d_{2 n+1}\left(K^{2}\right) \neq 0\right\} .
$$

(6.1) implies that

$$
c_{2 n}\left(\frac{1}{\rho}\right)=0 \quad \forall n \geq m+r+2 .
$$

Therefore there exists $s \in\{1, \ldots, 2(m+r+1)\}$ such that

$$
\frac{1}{\rho(\theta)}=c_{0}\left(\frac{1}{\rho}\right)+\sum_{p=-s}^{s} c_{p}\left(\frac{1}{\rho}\right) e^{i p \theta} \quad 0 \leq \theta<2 \pi,
$$

where $c_{s}(1 / \rho) \neq 0$. Since $c_{s}(1 / \rho) c_{2 m+1}(\rho)=c_{s+2 m+1}(1)=0$, we reach a contradiction and the lemma is proved.

Proof of Proposition 6.2. In Lemma 2.6 we have

$$
a=0, \quad b=8 \pi \psi^{2} \overline{c_{3}\left(\frac{1}{\rho}\right)},
$$

and for $k=2 p+1, p \geq 2$

$$
\begin{aligned}
& \beta_{1, k}=0 \\
& \alpha_{2, k}=(-1)^{p} \frac{4 \pi}{3}(p-1) p(p+1)(p+2) \overline{c_{k}\left(\frac{1}{\rho}\right)} \\
& \gamma_{1, k}=(-1)^{p} \frac{4 \pi}{3} p(p+1)\left(p^{2}+p+1\right) \psi^{2} \overline{c_{k}\left(\frac{1}{\rho}\right)} .
\end{aligned}
$$

By Lemma $6.1 k_{7}<\infty$. Then, using Lemma 2.6 with the above formulas, we deduce that $\mu$ satisfies a polynomial equation of degree $k_{7}$. Using Lemma 2.8 we conclude that there exist at most $k_{7}(\psi)$ different pairs of coefficients $\left(\lambda_{k}, \mu_{k}\right) \in$ $\mathbb{R}^{2}$ such that the Cauchy problem (1.5) has a solution.

Remark 6. In the setting of Proposition 6.2, condition (*) in Theorem 1.1 necessarily holds. Indeed suppose the contrary. Then we have

$$
\frac{1}{K(x)}=\frac{1}{K\left(x^{*}\right)}, \quad \forall x \in \partial \Omega .
$$

Since $\Omega$ is of constant width this implies that $\Omega$ is a disk, a contradiction with ii) in Definition 1.1. 
Definition 6.1. $\Omega \subset \mathbb{R}^{2}$ is said to be of class $\mathcal{C}^{\prime}$ if the following conditions hold:

i) $\Omega$ is a bounded convex open set and $\partial \Omega$ is a $C^{\infty}$ curve with positive curvature $K$.

ii) $d_{3}(1) \neq 0$.

iii) There exists $n \in \mathbb{Z}^{*}$ such that $d_{2 n}(1) \neq 0$.

Remark 7. Notice that $\mathcal{C} \subset \mathcal{C}^{\prime}$.

Proposition 6.3. Let $\Omega \subset \mathbb{R}^{2}$ be of class $\mathcal{C}^{\prime}$. Let $\psi$ be a non zero constant. Define

$$
k_{8}=2 \inf \left\{n \in \mathbb{N}^{*} ; d_{2 n}(1) \neq 0\right\}-1
$$

Then there exist at most $k_{8}$ different pairs of coefficients $\left(\lambda_{k}, \mu_{k}\right) \in \mathbb{R}^{2}$ such that the Cauchy problem (1.5) has a solution.

Proof. In Lemma 2.6 2) $a=0$. In Lemma 2.5 we have

$$
A=4 i \pi \psi \overline{c_{2}(h)} \quad \text { and } \quad B_{1,2 p}=(-1)^{p} 4 i \pi \psi \overline{c_{2 p}(h)} \quad \text { for } p \geq 2 .
$$

We deduce that $\mu$ satisfies a polynomial equation of degree $k_{8}$. Using Lemma 2.8 we conclude that there exist at most $k_{8}$ different pairs of coefficients $\left(\lambda_{k}, \mu_{k}\right) \in$ $\mathbb{R}^{2}$ such that the Cauchy problem (1.5) has a solution.

Definition 6.2. $\Omega \subset \mathbb{R}^{2}$ is said to be of class $\mathcal{D}^{\prime}$ if the following conditions hold:

i) $\Omega$ is a bounded convex open set and $\partial \Omega$ is a $C^{\infty}$ curve with positive curvature $K$.

ii) $d_{2}(l) \neq 0$.

iii) $\left\{n \in \mathbb{N}^{*} ; d_{2 n}(1) \neq 0\right\} \neq \emptyset$ is finite.

Remark 8. Notice that, by Remark $4, \mathcal{D}^{\prime} \subset \mathcal{D}$.

Proposition 6.4. Let $\Omega \subset \mathbb{R}^{2}$ be of class $\mathcal{D}^{\prime}$. Let $\psi$ be a non zero constant. Then

$$
k_{9}(\psi)=2 \inf \left\{n \geq 2 ; B_{1,2 n} d_{2}(l) \neq(-1)^{n} A d_{2 n}(l)\right\}-1<\infty
$$


or

$$
k_{10}=2 \inf \left\{n \in \mathbb{N}^{*} ; d_{2 n+1}(1) \neq 0\right\}-1<\infty,
$$

and $k_{9}(\psi)<\infty$ when $c_{2}(h)=0$. Let

$$
k_{0}= \begin{cases}\min \left(k_{9}(\psi), k_{10}\right) & \text { if } c_{2}(h) \neq 0 \\ k_{9}(\psi) & \text { if } c_{2}(h)=0\end{cases}
$$

Then there exist at most $k_{0}$ different pairs of coefficients $\left(\lambda_{k}, \mu_{k}\right) \in \mathbb{R}^{2}$ such that the Cauchy problem (1.5) has a solution.

\section{Proof. We can write}

$$
h(\theta)=\sum_{n=-q}^{q} c_{2 n}(h) e^{2 i n \theta}+\sum_{n=-\infty}^{\infty} c_{2 n+1}(h) e^{(2 n+1) i \theta}, \quad 0 \leq \theta<2 \pi,
$$

where $q \geq 1$ and $c_{2 q}(h) \neq 0$. (6.2) holds. Since $d_{2}(l) \neq 0$, Lemma 2.52 ) implies that $\lambda$ is a polynomial of degree 2 in $\mu$. If $c_{2}(h)=0$, then $q \geq 2$ and $k_{9}(\psi)<\infty$ since $B_{1,2 q} \neq 0$. With the help of Lemma 2.5 we deduce that $\mu$ satisfies a polynomial equation of degree $k_{9}(\psi)$. Using Lemma 2.10 we conclude that there exist at most $k_{9}(\psi)$ different pairs of coefficients $\left(\lambda_{k}, \mu_{k}\right) \in \mathbb{R}^{2}$ such that the Cauchy problem (1.5) has a solution. Now suppose that $c_{2}(h) \neq 0$. If $d_{2 n+1}(1)=0$ for $n \in \mathbb{Z}$ we have $d_{4 q}(l)=2 i q\left(1-4 q^{2}\right) \overline{c_{2 q}(h)^{2}} \neq 0$ and $B_{1,4 q}=0$, then $k_{9}(\psi)<\infty$ and we conclude as before. Now suppose that there exists $n \in \mathbb{N}$ such that $d_{2 n+1}(1) \neq 0$. Since $d_{1}(1)=0, n \geq 1$. Then $k_{10}<\infty$. In Lemma 2.6 we have $a=0$ and $\beta_{1,2 p+1}=0$ for $p \geq 2$. Using Lemmas 2.52 ) and 2.62 ) and 3) we deduce that $\mu$ satisfies a polynomial equation of degree $k_{10}$. With the help of Lemma 2.10 we conclude that there exist at most $k_{10}$ different pairs of coefficients $\left(\lambda_{k}, \mu_{k}\right) \in \mathbb{R}^{2}$ such that the Cauchy problem (1.5) has a solution.

\section{$\S 7 . \quad$ Examples}

We conclude this paper with some examples.

Example 1. Let $m \in \mathbb{N}^{*}$ and $a, a_{j}, b_{j} \in \mathbb{R}, j=1, \ldots, m$. Define

$$
h(\theta)=a+\sum_{n=1}^{m} a_{n} \cos (2 n+1) \theta+b_{n} \sin (2 n+1) \theta, \quad 0 \leq \theta<2 \pi,
$$


with $\left|a_{1}\right|+\left|b_{1}\right|>0$ and

$$
a>4 \sum_{n=1}^{m} n(n+1)\left(\left|a_{n}\right|+\left|b_{n}\right|\right) .
$$

$h$ is of class $C^{\infty}$, and has period $2 \pi$ and

$\rho(\theta)=a-4 \sum_{n=1}^{m} n(n+1)\left(a_{n} \cos (2 n+1) \theta+b_{n} \sin (2 n+1) \theta\right)>0, \quad 0 \leq \theta<2 \pi$.

Then $h$ must be the support function of a convex set $\Omega$. Clearly $\Omega$ is of class $\mathcal{B}$.

Example 2. Let $a, b, c \in \mathbb{R}^{*}$ be such that $a>3|b|+8|c|$. Define

$$
h(\theta)=a+b \cos 2 \theta+c \cos 3 \theta, \quad 0 \leq \theta<2 \pi .
$$

$h$ is of class $C^{\infty}$, and has period $2 \pi$ and

$$
\rho(\theta)=a-3 b \cos 2 \theta-8 c \cos 3 \theta>0, \quad 0 \leq \theta<2 \pi .
$$

Then $h$ must be the support function of a convex set $\Omega$. We can verify that $\Omega$ is of class $\mathcal{C} \cap \mathcal{D}^{\prime}$. Notice that $k_{8}=1$ in Proposition 6.3 and that $k_{0}=1$ in Proposition 6.4.

Example 3. Let $a>0$ and define

$$
h(\theta)=a+\frac{15+12 \cos \theta}{17-8 \cos 2 \theta}, \quad 0 \leq \theta<2 \pi .
$$

$h$ is of class $C^{\infty}$, and has period $2 \pi$. With the notations of Section 4 we have

$$
h_{1}(\theta)=a+\frac{15}{17-8 \cos 2 \theta}=a+\sum_{n=-\infty}^{+\infty} 2^{-2|n|} e^{2 i n \theta}, \quad 0 \leq \theta<2 \pi,
$$

and

$$
h_{2}(\theta)=\frac{12 \cos \theta}{17-8 \cos 2 \theta}=\sum_{n=-\infty}^{+\infty} 2^{-|2 n+1|} e^{(2 n+1) i \theta}, \quad 0 \leq \theta<2 \pi .
$$

For $a$ sufficiently large we easily show that

$$
\rho(\theta)>0, \quad 0 \leq \theta<2 \pi .
$$

Then $h$ must be the support function of a convex set $\Omega$. We can verify that $\Omega$ is of class $\mathcal{C} \cap \mathcal{D}$. 


\section{References}

[1] Berenstein, C. A., An inverse spectral theorem and its relation to the Pompeiu problem, J. Anal. Math., 37 (1980), 128-144.

[2] Blum, J., Numerical Simulation and Optimal Control in Plasma Physics, Wiley/ Gauthier-Villars, New York, 1989.

[3] Brown, L. and Kahane, J. P., A note on the Pompeiu problem for convex domains, Math. Ann., 259 (1982), 107-110.

[4] Dalmasso, R., A new result on the Pompeiu problem, Trans. Amer. Math. Soc., 352 (2000), 2723-2736.

[5] _ A note on the Schiffer conjecture, Hokkaido Math. J., 28 (1999), 373-383.

[6] , An inverse problem for an elliptic equation with an affine term, Math. Ann., 316 (2000), 771-792.

[7] Flanders, H., A proof of Minkowski's inequality for convex curves, Amer. Math. Monthly, 75 (1968), 581-593.

[8] Hurwitz, M. A., Quelques applications géométriques des séries de Fourier, Ann. Scuola Norm. Sup. Pisa, 19 (1902), 357-408.

[9] Kinderlehrer, D. and Nirenberg, L., Regularity in free boundary problems, Ann. Scuola Norm. Sup. Pisa, 4 (1977), 373-391.

[10] Serrin, J., A symmetry problem in potential theory, Arch. Rational Mech. Anal., 43 (1971), 304-318.

[11] Vogelius, M., An inverse problem for the equation $\Delta u=-c u-d$, Ann. Inst. Fourier, 44-4 (1994), 1181-1209. 